Check for updates

Cite this: Mater. Adv., 2021, 2,3300

Received 23rd January 2021, Accepted 27th March 2021

DOI: $10.1039 / \mathrm{d} 1 \mathrm{ma} 00058 \mathrm{f}$

rsc.li/materials-advances

\section{A novel PVDF hybrid membrane with excellent active-passive integrated antifouling and antibacterial properties based on a PDA guiding effect $\uparrow$}

\author{
Xing-Xing $\mathrm{Ni}$, Jian-Hua Li (D) and Lu-Ping Yu
}

A novel and versatile active-passive integrated strategy to integrate both antifouling and antibacterial properties into PVDF membranes was developed. PDA microspheres on modified membranes served as primers, and we proposed that a "PDA guiding effect" existed during the modification process. Specifically speaking, it was a guiding reduction effect from the PDA microspheres toward $\mathrm{Ag}^{+}$and a guiding generation effect toward L-cysteine that were separately in favor of the generation of AgNPs and cysteine flowers in cross-sections of the membranes. In addition, the active-passive integrated strategy presented in this research simultaneously includes both an anti-adhesion approach for reducing the initial adsorption of organisms and an anti-bacterial approach for suppressing the activities of attached organisms on the membranes. Conventional filtration experiments clearly demonstrated that the zwitterionic characteristics of L-cysteine made it possible to greatly reduce the initial adhesion of foulants and the deposition of dead bacteria on the membranes. Various bacteria-based experiments proved that AgNPs were effective at preventing the growth, proliferation, and colonization of microorganisms, as well as the accompanying membrane biofouling after long-term operation. Furthermore, self-driven filtration experiments, natural organic matter (NOM) solution filtration experiments, and protein adsorption tests were also carried out to evaluate the practical applications of the modified membranes. In this research, the PDA guiding effect was directed toward inorganic AgNPs and organic L-cysteine. This effect has great possibilities to be extended to other functional materials and to be broadly applied in the construction of diverse functionalized surfaces.

\section{Introduction}

Recently, membrane separation has become one of the most popular separation technologies because of its simple operation process, low energy consumption, high efficiency, and other outstanding merits. ${ }^{1,2}$ It has been widely used in many fields, such as wastewater treatment, ${ }^{3}$ desalination, ${ }^{4}$ food processing, ${ }^{5}$ biopharmaceuticals, ${ }^{6}$ environmental engineering, ${ }^{7}$ and so on. However, membrane fouling is considered a major obstacle that hinders the widespread application of membrane separation technology. In particular, membrane biofouling is very difficult to overcome due to the self-replicating nature of organisms, and it has strong negative effects on the operational sustainability and cost-efficiency of membrane separation. ${ }^{8,9}$ To mitigate membrane biofouling, current efforts have been directed toward the modification of membranes based on two main approaches:

College of Biological Science and Engineering, Fuzhou University, Fuzhou 350001,

P. R. China. E-mail:jhli_2005@163.com

$\dagger$ Electronic supplementary information (ESI) available. See DOI: 10.1039/d1ma00058f an anti-adhesion approach for reducing the initial adsorption of organisms; and an anti-bacterial approach for suppressing the activities of attached organisms on the membranes. ${ }^{10,11}$ However, it is still a challenge to find a facile and versatile method to integrate both these two main approaches for achieving excellent anti-biofouling properties.

Inspired by the mussel adhesive proteins excreted by marine mussels, a large number of researchers have focused on the exploration of biomimetic dopamine. As has been reported, the adherent polydopamine (PDA) can be formed on virtually all types of material surfaces via spontaneous oxidative self-polymerization in aqueous solutions. ${ }^{12-14}$ Specifically, PDA has stimulated extensive research relating to the functionalization of membranes for the great enhancement of hydrophilicity and antifouling performance. Moreover, the catechol groups on PDA make further incorporation with other functional substances possible. However, in most cases, PDA often plays an intermediary role and functions as a kind of simple surface modification chemical or additive during blending modification. The PDA guiding effect during modification processes has not been reported on to date. We first 
propose that PDA microspheres exhibit a guiding reduction effect on metal ions and a guiding generation effect on organic biomolecules that are separately in favor of the generation of metal nanoparticles and biomolecules in the interiors of substrates. In such cases, metal nanoparticles and organic biomolecules could be immobilized on the entire structure of a PDA-modified substrate and, therefore, the substrate could be endowed with better properties.

Silver nanoparticles (AgNPs) are widely used in various fields, such as catalysis, molecular recognition, biological labeling, and so on. ${ }^{15,16}$ In particular, AgNPs have attracted great attention as the most popular antibacterial agents for mitigating membrane biofouling due to their strong and broad-spectrum antibacterial properties and low cytotoxicity. ${ }^{17,18}$ With regard to the modification of membranes with AgNPs, both surface-coated modification and blending modification methods have been researched and gained ground. The surface-coated modification of membranes using AgNP deposition has been investigated for improving biofouling resistance. Dong et al. reported a green approach for immobilizing AgNPs on an RO membrane surface with the assistance of an ultrathin TA-Fe-PEI precursor layer. ${ }^{19}$ Biswas et al. developed an Ag-SPES hybrid membrane via the surface-impregnation of AgNPs onto PES membranes, achieving good antibacterial properties. ${ }^{20}$ However, in most cases involving a surface-coated approach, the formed AgNPs are loosely bound on the membranes and are easily washed away. Also, strong reducing agents are always needed for the generation of AgNPs. Blending modification with AgNPs directly incorporates AgNPs or functionalized AgNPs into the casting solution to fabricate blend membranes. Behboudi et al. prepared PVC-based hollow fiber ultrafiltration membranes embedded with pristine and modified AgNPs via a wet spinning method. ${ }^{21} \mathrm{Xu}$ et al. produced PSF blended ultrafiltration membranes using $\mathrm{Ag}-\mathrm{Cu}_{2} \mathrm{O}$ hybrid nanowires to achieve long-term antibacterial properties. ${ }^{22}$ However, blending modification requires either AgNPs or membrane substrates that are functionalized to promote strong bonding. Also, the agglomeration of AgNPs is prone to occur during the formation process of the membrane. Furthermore, in most cases, surface-coated and blending modification endow only membrane surfaces with better properties, not cross-sections. As a result, the excellent antibacterial properties of AgNPs cannot be fully realized. Considering all these factors, we believe that there is an exigent demand to develop a green, facile, and versatile strategy for immobilizing AgNPs onto the overall membrane structure.

Zwitterionic materials have been well proven to be highly promising antifouling compounds, due to their super antifouling properties for resisting nonspecific protein adsorption and bacterial adhesion. ${ }^{23,24}$ L-Cysteine contains a thiol group $(-\mathrm{SH})$ in its side chain and a pair of ionogenic amino $\left(-\mathrm{NH}_{2}\right)$ and carboxyl $(-\mathrm{COOH})$ groups, and it is thought of as a natural source of zwitterions. In addition, L-cysteine is a low-cost and widely available material, and it can conjugate additional molecules through amine or carboxyl functional groups, as well as through simple and convenient reactions involving thiol chemistry. ${ }^{25,26}$ To date, L-cysteine has been extensively researched and applied in many fields, such as biopharmaceuticals, food additives, wastewater treatment, and so on. ${ }^{27,28}$ L-Cysteine has been applied to the modification of membranes in recent decades and research has been reported. Azari et al. modified commercial TFC aromatic polyamide RO membranes via the grafting of L-cysteine, and the fouling resistance of the modified membranes was improved obviously. ${ }^{29}$ Liao et al. allowed protonated L-cysteine to be entrapped in a Nafion (Nf) membrane to form a Nf-Cys composite membrane, and the prepared immunosensor showed an excellent current response toward CEA. ${ }^{30}$ However, large plate-like L-cysteine is always grafted onto the membrane surface, and it is difficult to achieve the full coverage of $\mathrm{L}$-cysteine on the membrane cross-section.

In this paper, we report a novel and versatile active-passive integrated strategy to integrate both antifouling and antibacterial properties into PVDF membranes. A commercial PVDF membrane was first modified via the in situ generation of bio-inspired PDA microspheres; details of this research are presented in our previous work. ${ }^{31}$ Then, AgNPs were immobilized on the PVDF/PDA hybrid membrane via the reduction of silver nitrate aqueous solution due to the reducing properties of the catechol groups of PDA. Zwitterionic L-cysteine was further grafted onto the modified membranes via conducting a Michael addition reaction at $60{ }^{\circ} \mathrm{C}$. The PDA microspheres on the modified membranes served as primers. We proposed that a PDA guiding effect exists during the modification process. A guiding reduction effect from the PDA microspheres on $\mathrm{Ag}^{+}$and a guiding generation effect on L-cysteine were separately in favor of the generation of AgNPs and cysteine flowers in the membrane cross-section. Owing to this PDA guiding effect, the antibacterial and antifouling characteristics of the overall membrane structure can be enhanced greatly. The effects of modification on the surface and cross-section morphologies, hydrophilicity, permeability, antifouling properties, antibacterial properties, and long-term stability of the various prepared hybrid membranes were investigated in detail. The PDA guiding effect was focused on inorganic AgNPs and organic L-cysteine in this research, and it has great potential to be extended to other functional materials and broadly applied to the construction of diverse functionalized surfaces.

\section{Experimental}

\subsection{Materials and reagents}

Polyvinylidene fluoride (PVDF) microfiltration membranes were obtained from Membrane Solutions LLC Co. Ltd (Germany). The round membranes have a mean pore size of $0.45 \mu \mathrm{m}$ and a diameter of $50 \mathrm{~mm}$. Dopamine hydrochloride and silver nitrate $\left(\mathrm{AgNO}_{3}\right)$ were purchased from Sigma-Aldrich. L-Cysteine (Cys), bovine serum albumin (BSA; purity $\geq 98 \%$ ), humic acid (HA), and sodium alginate (SA) were supplied by Aladdin Reagent Co. Other reagents, such as tris(hydroxymethyl)aminomethane (Tris), 68\% nitric acid $\left(\mathrm{HNO}_{3}\right)$, and ethanol, were obtained from Sinopharm Chemical Reagent Co. (China). Escherichia coli (E. coli) and Staphylococcus aureus (S. aureus) were obtained from the inquiry network for microbial strains of China. All chemicals and reagents were of analytical grade. Deionized water was used in all experiments. 


\subsection{Preparation of the hybrid membranes}

2.2.1. Preparation of the PVDF/PDA-AgNPs hybrid membranes. PVDF/PDA hybrid membranes were prepared according to our previous work. ${ }^{31}$ To generate AgNPs on the membrane, the prepared PVDF/PDA hybrid membranes were directly incubated in $100 \mathrm{~mL}$ of silver nitrate aqueous solution under gentle stirring for $24 \mathrm{~h}$ at room temperature. The concentrations of silver nitrate solution were varied $(5,20$, and $40 \mathrm{mM})$ to investigate the effects of concentration on the generation of AgNPs on the membranes. After the reaction, the modified membranes were taken out and rinsed with deionized water to remove any non-firmly immobilized silver nanoparticles. The dried PVDF/PDA-AgNPs hybrid membranes were used for further characterization and modification. The PVDF/PDA-AgNPs hybrid membranes made with 5, 20, and $40 \mathrm{mM}$ silver nitrate solution are referred to as M2-5, M2-20, and M2-40, respectively, while the pristine PVDF membrane is referred to as M0, and the PVDF/PDA hybrid membrane prepared after being stirred for $24 \mathrm{~h}$ is referred to as M1.

2.2.2. Preparation of the PVDF/PDA-AgNPs-Cys hybrid membranes. L-Cysteine was covalently attached to the prepared hybrid membranes through thiol chemistry via a Michael addition reaction. The PVDF/PDA-AgNPs hybrid membranes were immersed into $200 \mathrm{~mL}$ of $\mathrm{L}$-cysteine aqueous solution and stirred gently for $24 \mathrm{~h}$. The reaction was carried out at $60{ }^{\circ} \mathrm{C}$ under oil-bath conditions. The concentrations of L-cysteine aqueous solution were varied $\left(1,2,3\right.$, and $\left.4 \mathrm{mg} \mathrm{mL}{ }^{-1}\right)$. After cooling down to room temperature, the obtained PVDF/PDA-AgNPs-Cys hybrid membranes were rinsed with deionized water to remove any residual L-cysteine. Then the membranes were dried and used for further characterization. The PVDF/PDA-AgNPs-Cys hybrid membranes made with $1,2,3$, and $4 \mathrm{mg} \mathrm{mL}^{-1}$ L-cysteine solution are referred to as M3-1, M3-2, M3-3, and M3-4, respectively.

\subsection{Membrane characterization}

The morphologies of the hybrid membranes were observed via field-emission scanning electronic microscopy (FESEM, Nova Nano SEM 230, FEI Co. Ltd, USA). The chemical compositions were characterized via X-ray photoelectron spectroscopy (XPS, ESCALAB 250, Thermo Scientific, USA) and attenuated total reflectanceFourier-transform infrared spectroscopy (ATR-FTIR, Thermo Nicolet 360 , Nicolet, USA). To evaluate the hydrophilicity of the membranes, water contact angle measurements were carried out via the sessile drop method using a contact angle analyzer (WCA, Dataphysics OCA20, Dataphysics Co., Germany), and more than five measurements on different areas of each membrane were performed. The changes in water contact angle were also recorded for a certain length of time. The surface zeta potentials of the different membranes were measured using an electrokinetic analyzer (NanoPlus 3, Micromeritics Instrument Corp., USA) via the streaming potential method based on the Helmholtz-Smoluchowski approach.

\subsection{Permeability and antifouling properties of the hybrid membranes}

2.4.1. Filtration and self-driven filtration experiments. The permeability and antifouling properties of the hybrid membranes were evaluated based on filtration experiments. The experiments were conducted using dead-end filtration apparatus according to a reported procedure ${ }^{32}$ and $1.0 \mathrm{~g} \mathrm{~L}^{-1}$ BSA aqueous solution served as the model foulant. The experiments were carried out at a constant pressure of $0.1 \mathrm{MPa}$ after precompaction of the membranes for $1 \mathrm{~h}$ at $0.2 \mathrm{MPa}$. First, the filtration of water was conducted for $70 \mathrm{~min}$ and the stable value of water flux was recorded as $J_{\mathrm{w} 1}\left(\mathrm{~L} \mathrm{~m}^{-2} \mathrm{~h}^{-1}\right)$. Then the filtration of $1.0 \mathrm{~g} \mathrm{~L}^{-1}$ BSA solution was also conducted for another $70 \mathrm{~min}$ and the stable value of BSA solution flux was recorded as $J_{\mathrm{p} 1}\left(\mathrm{~L} \mathrm{~m}^{-2} \mathrm{~h}^{-1}\right)$. After washing in deionized water for $4 \mathrm{~h}$, the cleaned membrane was further used for the filtration of water and BSA solution. The subsequent stable water flux and BSA solution flux values were separately recorded as $J_{\mathrm{w} 2}\left(\mathrm{~L} \mathrm{~m}^{-2} \mathrm{~h}^{-1}\right)$ and $J_{\mathrm{p} 2}\left(\mathrm{~L} \mathrm{~m}^{-2} \mathrm{~h}^{-1}\right) \cdot{ }^{33-35}$

The water flux $\left(J_{\mathrm{w}}, \mathrm{L} \mathrm{m}^{-2} \mathrm{~h}^{-1}\right)$ and BSA solution flux $\left(J_{\mathrm{p}}, \mathrm{L} \mathrm{m}^{-2} \mathrm{~h}^{-1}\right)$ of the membranes were calculated using eqn (1) and (2):

$$
\begin{gathered}
J_{\mathrm{W}}=\frac{V_{\mathrm{W}}}{A \Delta t} \\
J_{\mathrm{P}}=\frac{V_{\mathrm{P}}}{A \Delta t}
\end{gathered}
$$

where $V_{\mathrm{W}}(\mathrm{L})$ and $V_{\mathrm{P}}(\mathrm{L})$ represent the volumes of permeated water and BSA solution, respectively, $\Delta t(\mathrm{~h})$ represents the permeation time, and $A\left(\mathrm{~m}^{2}\right)$ represents the effective filtration area of the membrane (here $A=5.0 \times 10^{-4} \mathrm{~m}^{2}$ ).

The BSA rejection $(R)$ was calculated according to eqn (3):

$$
R(\%)=1-\frac{C_{\mathrm{P}}}{C_{\mathrm{F}}} \times 100
$$

where $C_{\mathrm{P}}\left(\mathrm{mg} \mathrm{mL}^{-1}\right)$ and $C_{\mathrm{F}}\left(\mathrm{mg} \mathrm{mL}^{-1}\right)$ represent the BSA concentrations in the penetrating solution and feed solution, respectively. A UV-vis spectrophotometer (UV-2550, Shimadzu, Japan) was used to measure the BSA concentration at a wavelength of $280 \mathrm{~nm}$.

The water flux recovery $\left(\mathrm{FR}_{\mathrm{W}}\right)$ and flux loss caused by total protein fouling $\left(R_{\mathrm{t}}\right)$ were calculated using eqn (4) and (5):

$$
\begin{aligned}
& \mathrm{FR}_{\mathrm{W}}(\%)=\left(\frac{J_{\mathrm{W} 2}}{J_{\mathrm{W} 1}}\right) \times 100 \\
& R_{\mathrm{t}}(\%)=\left(\frac{J_{\mathrm{W} 1}-J_{\mathrm{P}}}{J_{\mathrm{W} 1}}\right) \times 100
\end{aligned}
$$

The flux loss values resulting from both reversible and irreversible protein fouling $\left(R_{\mathrm{r}}\right.$ and $\left.R_{\mathrm{ir}}\right)$ were calculated via eqn (6) and (7):

$$
\begin{gathered}
R_{\mathrm{r}}(\%)=\left(\frac{J_{\mathrm{W} 2}-J_{\mathrm{P}}}{J_{\mathrm{W} 1}}\right) \times 100 \\
R_{\mathrm{ir}}(\%)=\left(\frac{J_{\mathrm{W} 1}-J_{\mathrm{W} 2}}{J_{\mathrm{W} 1}}\right) \times 100=100 \%-\mathrm{FR}_{\mathrm{W}}
\end{gathered}
$$

The self-driven filtration experiments were conducted using dead-end filtration apparatus. The apparatus was similar to a gravity-driven membrane (GDM) filtration system, ${ }^{36,37}$ and no external pressure or electricity supply was needed in the experiments. As reported, deionized water flowed from the feed tank 
to the membrane filtration cell, while the water level in the feed tank was kept constant based on an overflow system and periodical pumping from the storage tank. The permeated water from the membrane filtration cell was collected during the $4 \mathrm{~h}$ of filtration. The permeated water flux values $\left(J_{\mathrm{w}}, \mathrm{L} \mathrm{m}^{-2} \mathrm{~h}^{-1}\right)$ of the membranes were calculated according to eqn (1).

2.4.2. Natural organic matter (NOM) solution filtration experiments. The membranes were also tested via filtration experiments involving synthetic NOM solution to assess their practical application potential. The NOM solution was prepared according to reported works. ${ }^{38,39}$ Humic acid (HA), sodium alginate (SA), and bovine serum albumin (BSA) were chosen as the representative pollutants for humic substances, polysaccharides, and proteins, respectively. The raw concentrations of HA, SA, and BSA were $4.0,2.0$, and $4.0 \mathrm{mg} \mathrm{mL}^{-1}$, respectively, and the composite foulants were dissolved in deionized water. Moreover, $1 \mathrm{mM} \mathrm{CaCl}_{2}$ solution and $7 \mathrm{mM} \mathrm{NaCl}$ solution were used as background solutions. The synthetic NOM solution was filtered using the membranes at a constant pressure of $0.1 \mathrm{MPa}$ under a stirring speed of $400 \mathrm{rpm}$, and the permeation flux values were recorded.

The NOM solution flux values $\left(J_{\text {NOM }}, \mathrm{L} \mathrm{m}^{-2} \mathrm{~h}^{-1}\right)$ of the membranes were calculated according to eqn (8):

$$
J_{\mathrm{NOM}}=\frac{V_{\mathrm{NOM}}}{A \Delta t}
$$

where $V_{\text {NOM }}(\mathrm{L})$ represents the volume of permeated NOM solution, $\Delta t(\mathrm{~h})$ represents the permeation time, and $A\left(\mathrm{~m}^{2}\right)$ represents the effective filtration area of the membrane (here $A=5.0 \times 10^{-4} \mathrm{~m}^{2}$ ).

The total membrane resistance $\left(R_{\mathrm{T}}\right)$ was defined to evaluate the antifouling properties of the membranes toward NOM solution. It was calculated using eqn (9):

$$
R_{\mathrm{T}}(\%)=\left(\frac{\Delta P}{\mu J_{\mathrm{NOM}}}\right) \times 100
$$

where $\Delta P(\mathrm{MPa})$ represents the constant pressure during filtration, and $\mu$ (Pa s) represents the dynamic viscosity of the NOM solution.

2.4.3. Pollutant adsorption tests. The antifouling properties of the membranes were also evaluated via static adsorption tests. Bovine serum albumin (BSA) and humic acid (HA) were chosen as model protein and humic substances, respectively. The test sample concentrations were $0.4 \mathrm{~g} \mathrm{~L}^{-1}$ and $0.6 \mathrm{~g} \mathrm{~L}^{-1}$ for BSA and HA solution (dissolved in PBS buffer solution with $\mathrm{pH}=7.4$ ). The membrane samples were regularly shaped with effective areas of $0.6 \mathrm{~cm} \times 2 \mathrm{~cm}$. After being immersed and shaken in pollutant solution for $24 \mathrm{~h}$, the membrane samples were taken out and rinsed with PBS buffer solution several times to remove non-firmly attached BSA or HA. The mass $\left(\mu \mathrm{g} \mathrm{cm}^{-2}\right)$ of the adsorbed BSA or HA on the membrane was obtained via calculating the concentrations of the pollutant solution before and after testing. The concentrations of BSA and HA were measured with a UV-2550 spectrometer at wavelengths of $280 \mathrm{~nm}$ and $253 \mathrm{~nm}$, respectively.

\subsection{Antibacterial properties of the hybrid membranes}

2.5.1. Diffusion inhibition zone (DIZ) tests. The antibacterial properties of the membranes were studied using the Gram-positive bacteria $S$. aureus and the Gram-negative bacteria E. coli. E. coli and $S$. aureus colonies were incubated in Luria-Bertani (LB) medium at $37^{\circ} \mathrm{C}$. All the containers and membranes were sterilized before the tests. The concentration of bacterial suspension was diluted to $10^{6}$ CFU $\mathrm{mL}^{-1}$ and then $100 \mu \mathrm{L}$ of bacterial suspension was spread evenly on LB agar plates. Circular membrane samples that were $13.0 \mathrm{~mm}$ in diameter were then placed on the top of the plates, and the active layers of the membranes were in direct contact with the agar surface. After incubation at the optimal temperature (37 ${ }^{\circ} \mathrm{C}$ for $E$. coli and $30{ }^{\circ} \mathrm{C}$ for $S$. aureus) for $24 \mathrm{~h}$, diffusion inhibition zones were formed, and the antibacterial properties of the membranes could be evaluated via measuring the diameters of the inhibition zones. ${ }^{40}$

2.5.2. Colony forming unit (CFU) counting tests. The colony forming unit (CFU) counting method was also used to evaluate the antibacterial properties of the modified membranes. Bacterial suspensions of $E$. coli and $S$. aureus were prepared, and the concentrations were $10^{6} \mathrm{CFU} \mathrm{mL}^{-1}$. Circular membrane samples that were $13.0 \mathrm{~mm}$ in diameter were immersed into $10 \mathrm{~mL}$ of bacterial suspensions in tubes. After incubation at $37{ }^{\circ} \mathrm{C}$ under shaking (200 rpm) for $24 \mathrm{~h}$, the membranes were rinsed gently with deionized water several times, and these washing fluids were added to the bacterial suspensions. The prepared bacterial suspensions of M0 and M1 were serially diluted 100 times. Then, $100 \mu \mathrm{L}$ of the diluted bacterial suspensions of M0 and M1, and suspensions of the AgNP-modified membranes M2-X and M3-X without dilution were dispersed uniformly on LB agar plates, respectively. After incubation at the optimal temperature (37 ${ }^{\circ} \mathrm{C}$ for $E$. coli and $30{ }^{\circ} \mathrm{C}$ for $S$. aureus) for $24 \mathrm{~h}$, the bacteria colonies were counted. ${ }^{41}$ The percentage of bacterial viability was calculated using eqn (10):

$$
\text { Bacterial viability }(\%)=\left(\frac{N_{\mathrm{m}}}{N_{\mathrm{p}}}\right) \times 100
$$

where $N_{\mathrm{p}}$ and $N_{\mathrm{m}}$ represent the numbers of live bacteria in the prepared bacterial suspensions of pristine PVDF membrane and the modified membrane, respectively. The numbers of live bacteria in the initial undiluted suspensions of M0 and M1 could be calculated based on the products of the number of counted colonies and the dilution times. The experimental results were all obtained from three independent replicates.

2.5.3. Bacterial adhesion tests. The anti-adhesive properties of the membranes were evaluated based on bacterial adhesion tests, and E. coli was used as the model bacteria. A bacterial suspension was first prepared, and the concentration was diluted to $10^{8} \mathrm{CFU} \mathrm{mL}^{-1}$. Then membrane samples were placed in a 12-well cell culture plate and covered with the diluted bacterial suspension. After being incubated statically at $37{ }^{\circ} \mathrm{C}$ in a constant temperature incubator for $24 \mathrm{~h}$, the membrane samples were carefully rinsed with PBS buffer solution $(\mathrm{pH}=7.4)$ to remove non-firmly attached bacteria. After that, bacteria adhered on the membranes were fixed using 
$2.5 \mathrm{wt} \%$ glutaraldehyde solution for $30 \mathrm{~min}$. Then, the membranes were dehydrated with $10,30,50,70,90$, and $100 \%$ (v/v) ethanol/ water solution for $30 \mathrm{~min}$ in sequence and dried in a vacuum oven at $37{ }^{\circ} \mathrm{C}$ for 4 h. ${ }^{42,43} \mathrm{SEM}$ was utilized to observe the numbers of adherent bacteria on the membrane surfaces and to evaluate the anti-adhesive properties toward bacteria.

\subsection{Stability of the immobilized AgNPs on the membranes}

The stability and release behavior of the immobilized AgNPs on the membranes was evaluated via Ag leaching tests. Circular membranes that were $13 \mathrm{~mm}$ in radius were incubated in $20 \mathrm{~mL}$ of deionized water in tubes and were placed in a shaking bath at $30{ }^{\circ} \mathrm{C}$. After slight shaking for $24 \mathrm{~h}$, the solution was removed and used for analyzing the released amount of AgNPs. Then the tubes were refilled with another $20 \mathrm{~mL}$ of deionized water for prolonged release studies. The soaking solution was replaced at predetermined time intervals of $24 \mathrm{~h}$, and the leaching tests lasted for 30 days, with three samples prepared for each experimental group. The released amounts of AgNPs were measured via inductively coupled plasma mass spectrometry (ICP-MS, XSERIES 2, Thermo Fisher Scientific, Shanghai). $68 \%$ nitric acid was added to acidify the soaking solution before ICP-MS analysis. The concentration of dissolved silver was quantified via the analysis of ${ }^{107} \mathrm{Ag}$, with average values taken from three separate ICP-MS sweeps. ${ }^{44}$ The total mass of the released silver over 30 days was the sum of the measured masses from the daily samples. In addition, the antibacterial properties of the membranes after 30 days of $\mathrm{Ag}$ leaching tests were also re-evaluated.

\section{Results and discussion}

\subsection{Preparation and characterization of the hybrid membranes}

AgNPs and zwitterionic L-cysteine were immobilized on PVDF/ PDA hybrid membranes based on the reducing nature of the catechol groups of PDA and a Michael addition reaction, respectively. A schematic illustration of the preparation of the hybrid membranes is depicted in Fig. 1. The surface and crosssection morphologies of the membranes were observed via
SEM. As can be seen in Fig. 2A, D, and G, and Fig. S1 (ESI $\dagger$ ), AgNPs with an average diameter of about $30 \mathrm{~nm}$ were clearly observed on the entire structure of the PVDF/PDA-AgNPs hybrid membrane. A silver nitrate solution concentration of $40 \mathrm{mM}$ was optimal, and further modifications were carried out utilizing M2-40. In addition, it is worth appreciating that a great number of AgNPs were immobilized on PDA microspheres in the cross-section. During the reduction process, $\mathrm{Ag}^{+}$is preferentially absorbed onto the PDA layer due to the metal-binding abilities of the catechol groups of PDA, and this is then reduced to AgNPs due to the reducing nature of the catechol groups. Furthermore, the $\mathrm{O}$ sites and $\mathrm{N}$ sites of PDA can serve as anchors to guide the immobilization of AgNPs on PDA microspheres through metal coordination. ${ }^{45,46}$ Therefore, we propose that it is the guiding reduction effect of PDA microspheres on $\mathrm{Ag}^{+}$that greatly enhances the quantity and efficiency of the generation of AgNPs on the substrate interior. The existence of numerous AgNPs on the cross-section specifically imparted the membranes with excellent hydrophilicity, permeability, and antifouling performance during the filtration process.

The morphologies of the PVDF/PDA-AgNPs-Cys hybrid membranes are shown in Fig. 2 and Fig. S2 (ESI $\dagger$ ). It was obviously observed that the L-cysteine clusters presented flower shapes for M3-3 and M3-4, and these L-cysteine clusters were defined as cysteine flowers. These cysteine flowers on M3-3 and M3-4 displayed great 3D form without overwhelmingly covering the PDA microspheres and AgNPs, enabling PDA, AgNPs, and L-cysteine to all perform without interference. Interestingly, it could also be observed from Fig. $2 \mathrm{H}$ and $\mathrm{I}$ that the cysteine flowers existed not only on the membrane surface but also in the membrane pores of the cross-section. Amine or thiol groups containing biomolecules can be covalently immobilized onto PDA-modified substrates either via Michael addition or a Schiff base reaction at elevated temperature. ${ }^{47}$ PDA served as an anchor for guiding the immobilization of $\mathrm{L}$-cysteine on the PDA microspheremodified membrane surface and cross-section. Similarly, we believe that there exists a guiding generation effect of PDA microspheres toward L-cysteine, which is in favor of the generation of cysteine flowers on the substrate interior. The existence of abundant L-cysteine in the cross-section is beneficial for the generation of

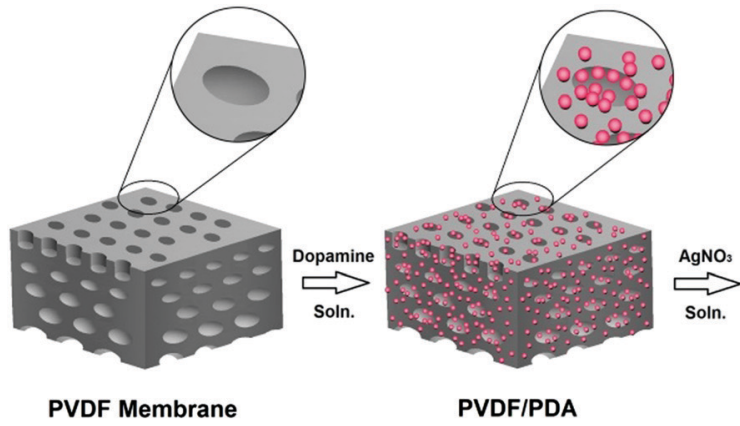

Polydopamine (PDA) microsphere

Ag nanoparticle

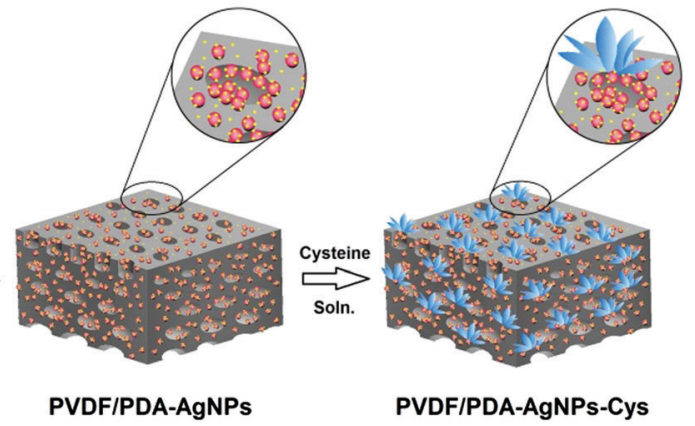

Cysteine flower

Fig. 1 Schematic illustration of the preparation of the hybrid membranes. 

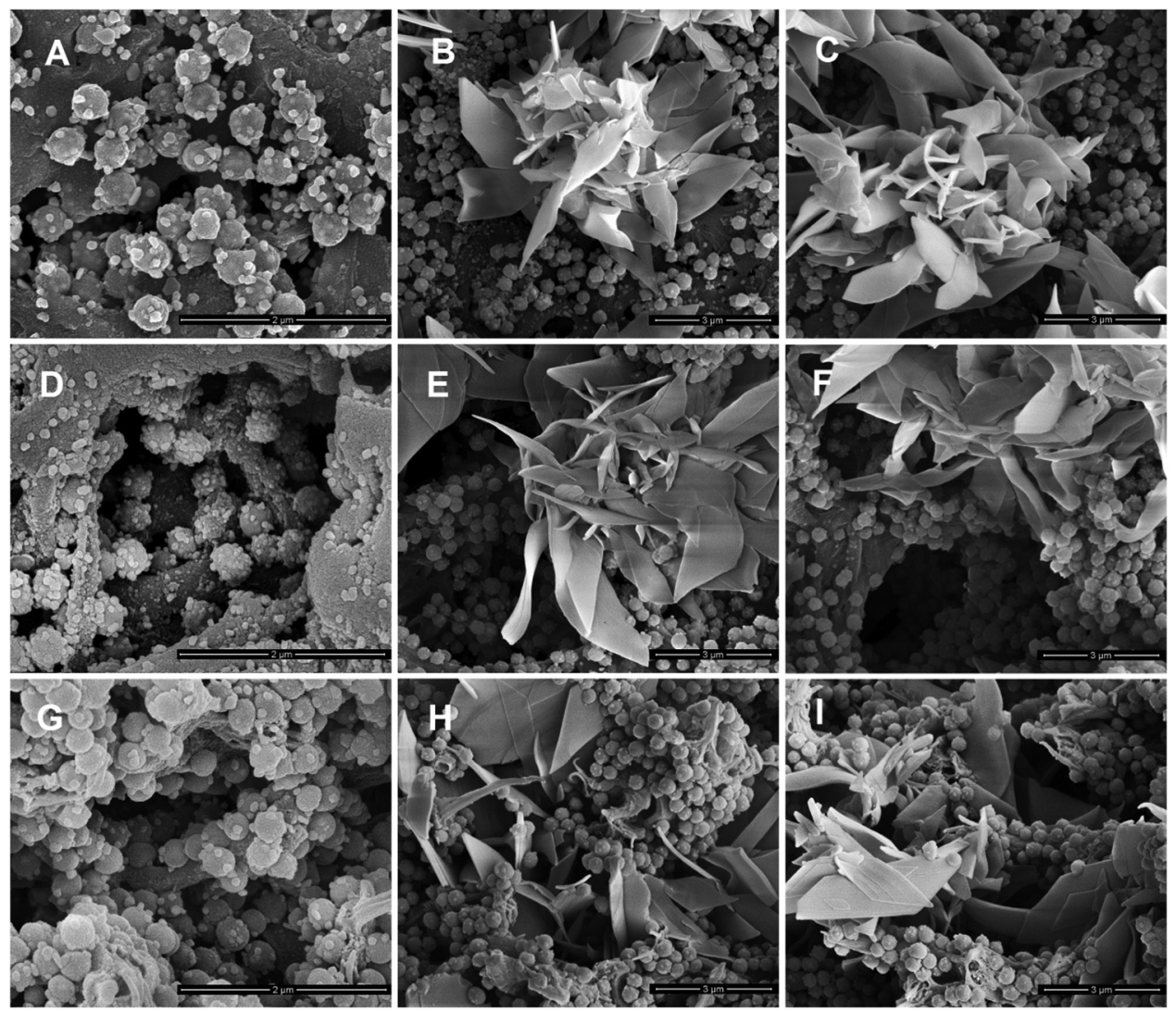

Fig. 2 The morphologies and structures of M2-40 (A, D and G), M3-3 (B, E and H), and M3-4 (C, F and I): enlarged SEM images of the upper surfaces (A$C)$, lower surfaces $(D-F)$, and cross-sections $(G-l)$ of the various membranes.

a hydration layer upon the membranes; this then imparts the hybrid membranes with superior antifouling performance during the filtration process. In addition, it was clearly seen that the presence of cysteine flowers did not result in the membrane pores being covered in large numbers, and the structures of the membranes pores in the cross-section were in good condition. The reason why L-cysteine exists in the shape of a flower is also discussed. The oppositely charged sidechain groups of amino acids, like the $-\mathrm{NH}_{3}{ }^{+}$and $-\mathrm{COO}^{-}$groups in L-cysteine solution could form ionic bonds. In addition, hydrogen bonds could also form between some functional groups, like $-\mathrm{NH}_{3}{ }^{+}$and $-\mathrm{SH}$. The ionic bonds and hydrogen bonds formed between different $\mathrm{L}$-cysteine molecules brought about a decrease in the fluidity of L-cysteine, which meant that platelike L-cysteine was more likely to aggregate and interconnect. As the concentration of L-cysteine increased, the amount of connected plate-like L-cysteine increased and, finally, it was immobilized on the membranes in the form of flowers. To further confirm the distribution of AgNPs and cysteine flowers on the membranes, SEM-EDS mapping was performed, and the data are shown in Fig. S3 (ESI $\dagger$ ). It can be observed that the AgNPs and cysteine flowers were successful immobilized and uniformly distributed over the whole membrane structure of M3-4.

Water contact angle measurements were conducted to evaluate the hydrophilicity of the membranes. As shown in Fig. 3A, the initial contact angle of the pristine PVDF membrane was found to be around $103^{\circ}$, showing the hydrophobic nature of the membrane. M1 showed a water contact angle of about $9.7^{\circ}$. After modification with AgNPs, the PVDF/PDA-AgNPs hybrid membranes displayed super-hydrophilic surfaces, and the initial contact angle of M2-40 was about $8.1^{\circ}$. This can be attributed to a combined effect of enhanced affinity for water and improved surface roughness after the introduction of hydrophilic AgNPs on the surface and cross-section of the membrane. After further incubating M2-40 in L-cysteine solution, the water contact angle displayed a great change from M3-1 to M3-4. The values were $25.4^{\circ}, 22.6^{\circ}, 9.4^{\circ}$, and $5.7^{\circ}$ for M3-1, M3-2, M3-3, and M3-4, respectively. The results indicated improved surface hydrophilicity upon increasing the concentration of $\mathrm{L}$-cysteine. The reasons for the water contact angle undergoing a great change are discussed. As shown in Fig. S2A, B and S2E, F (ESI $\dagger$ ), plate-like L-cysteine was dispersed on the surfaces, covering a great deal of the PDA microspheres and AgNPs. The hydrophilicity of a small amount of L-cysteine was poorer than PDA microspheres and AgNPs. As a result, the water contact angles of M3-1 and M3-2 relatively increased. When the concentration of $\mathrm{L}$-cysteine solution increased to $3 \mathrm{mg} \mathrm{mL}^{-1}$, cysteine flowers were formed and they covered the PDA microspheres and AgNPs in a dramatically reduced manner. This favored an improvement in the hydrophilicity of M3-3 and M3-4, and the surfaces of M3-3 and M3-4 even reached super-hydrophilicity. Fig. 3B showed the timedependence of the water contact angles of the membranes. 

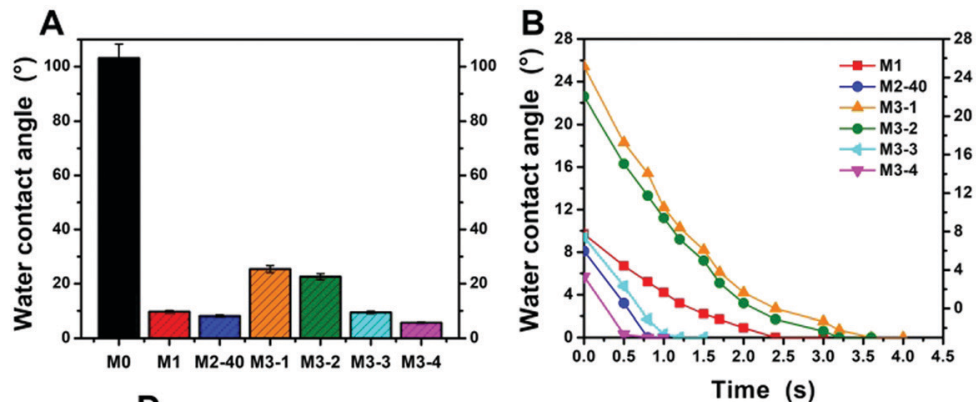

D

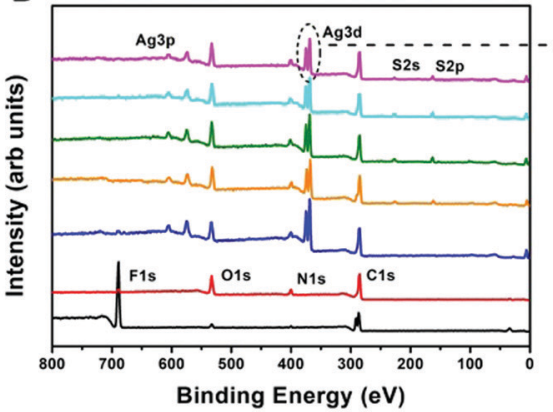

Time (s)

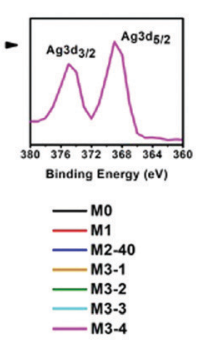

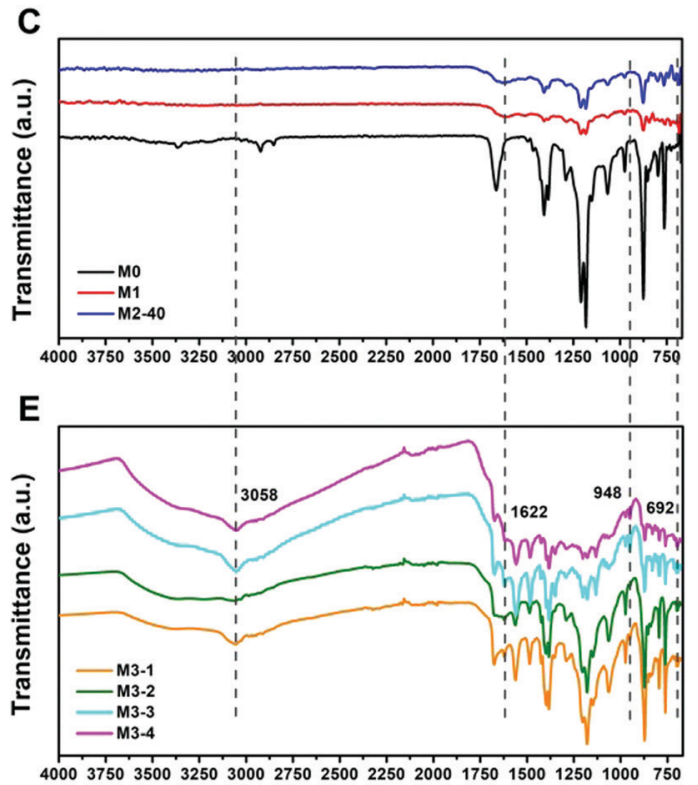

Wavenumbers $\left(\mathrm{cm}^{-1}\right)$

Fig. 3 The hydrophilicity and permeability properties of the various membranes: the initial water contact angles (A), and water contact angle versus drop time plots (B); ATR-FTIR spectra of the various membranes (C and E); and XPS survey spectra of the various membranes (D).

According to previous research, the water contact angle of the pristine PVDF membrane remained at about $101^{\circ}$ for $120 \mathrm{~s}$. However, the contact angles of all the modified membranes decreased obviously with increasing time, especially M2-40, M3-3, and M3-4. It was observed that the water contact angle of M2-40 dropped from 8.1 to $0^{\circ}$ in $0.8 \mathrm{~s}$, while M3-3 and M3-4 dropped from $9.4^{\circ}$ to $0^{\circ}$ in $1.1 \mathrm{~s}$ and from $5.7^{\circ}$ to $0^{\circ}$ in $0.6 \mathrm{~s}$, respectively. This rapid decrease in the water contact angles of the PVDF/PDA-AgNPs-Cys hybrid membranes implied that the cysteine flowers do not have a significant negative effect on permeability and wettability. The surfaces of M3-3 and M3-4 can simultaneously reach super-hydrophilicity and show outstanding permeability.

To confirm the successful functional modification of the pristine PVDF membrane, ATR-FTIR was performed to analyze the chemical compositions of the membranes, as shown in Fig. $3 \mathrm{C}$ and E. Compared to the pristine PVDF membrane, the spectrum of the PVDF/PDA hybrid membrane showed new clear peaks from 1650 to $1510 \mathrm{~cm}^{-1}$, which were attributed to the superposition of phenylic $\mathrm{C}=\mathrm{C}$ stretching vibrations, $\mathrm{N}-\mathrm{H}$ bending vibrations, and $\mathrm{N}-\mathrm{H}$ shearing vibrations. The new broad peaks between 3650 and $3025 \mathrm{~cm}^{-1}$ appearing for M1 were ascribed to $\mathrm{O}-\mathrm{H}$ stretching vibrations and $\mathrm{N}-\mathrm{H}$ stretching vibrations, suggesting the successful introduction of PDA microspheres onto the PVDF membrane. However, no noticeable changes were observed in the spectrum of M2-40 due to the fact that no new functional groups were formed during the reduction of AgNPs. For the L-cysteine modified membranes, the spectra all displayed a distinct new peak at around $692 \mathrm{~cm}^{-1}$, which was assigned to the $\mathrm{C}-\mathrm{S}$ stretching vibrations of $\mathrm{L}$-cysteine. In addition, new peaks were observed at approximately 948 and $1622 \mathrm{~cm}^{-1}$, which can be attributed to $\mathrm{O}-\mathrm{H}$ bending vibrations and $\mathrm{C}=\mathrm{O}$ stretching vibrations from the carboxyl functional group. Moreover, the spectra showed an intense peak at around $3058 \mathrm{~cm}^{-1}$ corresponding to $\mathrm{N}-\mathrm{H}$ stretching vibrations. All these spectral results for the L-cysteine-modified membranes indicated that $\mathrm{L}$-cysteine was successfully introduced onto the membrane. Generally, L-cysteine is said to exhibit characteristic peaks from 2600 to $2550 \mathrm{~cm}^{-1}$, which can be attributed to $-\mathrm{SH}$ stretching vibrations. However, these characteristic peaks were not observed in the M3-X spectra. The absence of -SH stretching vibrations can probably be attributed to the Michael addition reaction between the -SH bonds and PDA microspheres, as well as covalent interactions between the - $\mathrm{SH}$ bonds and AgNPs to form metal-thiol conjugations.

XPS analysis was utilized to determine the elemental compositions in the near-surface regions of the membranes, and the results are shown in Fig. 3D. It can be seen that the spectrum of the pristine PVDF membrane showed two peaks at binding energy (BE) values of 285.4 and $686.3 \mathrm{eV}$, which separately represent the $\mathrm{C}$ 1s and $\mathrm{F}$ 1s regions of the PVDF matrix. Two new peaks at 532.1 and $399.5 \mathrm{eV}$ were observed in the spectrum of M1. They were assigned to the $\mathrm{O} 1 \mathrm{~s}$ and $\mathrm{N} 1 \mathrm{~s}$ regions due to PDA components in the membrane. The spectrum of M2-40 clearly revealed the presence of silver atoms with $\mathrm{Ag} 3 \mathrm{~d}_{5 / 2}$ and $\mathrm{Ag} 3 \mathrm{~d}_{3 / 2}$ BEs at around 368.7 and $374.8 \mathrm{eV}$, respectively. These two new peaks were attributed to silver species, further confirming the successful generation of AgNPs on the PVDF/ PDA-AgNPs hybrid membranes. Additionally, the silver atomic percentage increased upon increasing the concentration of silver ions, and the M2-40 surface reached as high as $15.88 \%$, as shown in Table 1. After modification with L-cysteine, the spectra of M3-X all exhibited two new peaks at BEs of 163.6 and $228.9 \mathrm{eV}$, which respectively represented the S $2 \mathrm{p}$ and S $2 \mathrm{~s}$ regions of L-cysteine. The appearance of sulfur peaks confirmed 
Table 1 The surface elemental compositions of the various membranes

\begin{tabular}{|c|c|c|c|c|c|c|}
\hline \multirow[b]{2}{*}{ Membrane } & \multicolumn{6}{|c|}{ Element weight \% } \\
\hline & $\mathrm{C}$ & F & $\mathrm{O}$ & $\mathrm{N}$ & $\mathrm{Ag}$ & $\mathrm{S}$ \\
\hline M0 & 46.98 & 49.33 & 3.69 & - & - & - \\
\hline M1 & 68.17 & 2.21 & 22.50 & 7.12 & - & - \\
\hline M2-5 & 69.22 & 5.74 & 15.95 & 6.03 & 3.05 & - \\
\hline M2-20 & 71.14 & 2.91 & 16.37 & 5.84 & 3.74 & - \\
\hline M2-40 & 60.48 & 1.71 & 16.33 & 5.59 & 15.88 & - \\
\hline M3-1 & 66.59 & - & 15.85 & 7.38 & 5.47 & 4.71 \\
\hline M3-2 & 63.19 & - & 16.20 & 7.66 & 5.96 & 6.99 \\
\hline M3-3 & 62.00 & - & 16.15 & 7.84 & 5.30 & 8.70 \\
\hline M3-4 & 62.62 & - & 15.43 & 6.99 & 5.35 & 9.61 \\
\hline
\end{tabular}

that L-cysteine was indeed functionalized onto the membrane surfaces. In addition, an increasing sulfur atomic percentage was observed from M3-1 to M3-4, indicating the increased loading of $\mathrm{L}$-cysteine as the concentration of $\mathrm{L}$-cysteine solution was increased.

\subsection{Permeability and antifouling properties of the hybrid membranes}

The permeability and antifouling properties of the functionalized membranes were evaluated through multicycle filtration experiments using BSA as a model protein. Fig. 4A and $\mathrm{C}$ show the water flux and BSA flux during three filtration cycles, and Fig. $4 \mathrm{~B}$ exhibits the stable flux and the water flux recovery $\left(\mathrm{FR}_{\mathrm{W}}\right)$. In the first filtration cycle, the stable water flux values of the pristine PVDF membrane and M1 were $4080 \mathrm{~L} \mathrm{~m}^{-2} \mathrm{~h}^{-1}$ and $2760 \mathrm{~L} \mathrm{~m}^{-2} \mathrm{~h}^{-1}$, respectively. It was surprising that M2-40 and M3-X all showed relatively high water-flux levels, and the stable values were $7248,4152,4524,8256$, and $5016 \mathrm{~L} \mathrm{~m}^{-2} \mathrm{~h}^{-1}$, respectively, indicating improved permeability compared to the pristine PVDF membrane. The BSA flux values for all membranes experienced a dramatic decrease due to the deposition and adsorption of BSA on the membrane surface. However, M2-40 and M3-X still exhibited relatively high BSA flux levels. After simple physical cleaning with deionized water, the $\mathrm{FR}_{\mathrm{W}}$ value of the pristine PVDF membrane was merely $50.2 \%$ due to the poor antifouling properties, while all the modified membranes achieved more than $80 \%$ water flux recovery. Similarly, high water-flux, BSA-flux, and $\mathrm{FR}_{\mathrm{W}}$ values for all modified membranes were observed in the following two filtration cycles. The water flux values were more than ten times that of the pristine PVDF membrane, and the highest $\mathrm{FR}_{\mathrm{W}}$ value reached $91.0 \%$ in the case of M3-4. All these results indicated the excellent permeability and antifouling properties of the modified membranes, especially M2-40 and M3-3. To further quantify the antifouling properties of the membranes, the BSA rejection $(R)$, the degree of flux loss resulting from total protein fouling $\left(R_{\mathrm{t}}\right)$, and the flux losses caused by both reversible and irreversible protein fouling $\left(R_{\mathrm{r}}\right.$ and $\left.R_{\mathrm{ir}}\right)$ were calculated according to the equations. As shown in Fig. 4D, the BSA rejection levels of the modified membranes were all higher than that of the pristine PVDF membrane; M2-40 and M3-X all achieved more than $90 \%$ BSA rejection. In addition, the highest values of $R_{\mathrm{t}}$ and $R_{\mathrm{ir}}$ for the pristine PVDF membrane were $84.4 \%$ and $52.4 \%$ during the three filtration cycles. However, decreased $R_{\mathrm{t}}$ and $R_{\mathrm{ir}}$ values were observed for the modified membranes, and the values were all below 15\% for M2-40 and M3-X, indicating that irreversible fouling caused by the adsorption of protein and the blockage of pores were both diminished. All these results clearly demonstrated that the antifouling properties were effectively improved through modification with PDA microspheres, AgNPs, and L-cysteine.

In this paper, we focused on researching an active-passive integrated strategy to integrate both antifouling and antibacterial properties into modified membranes. The active-passive integrated
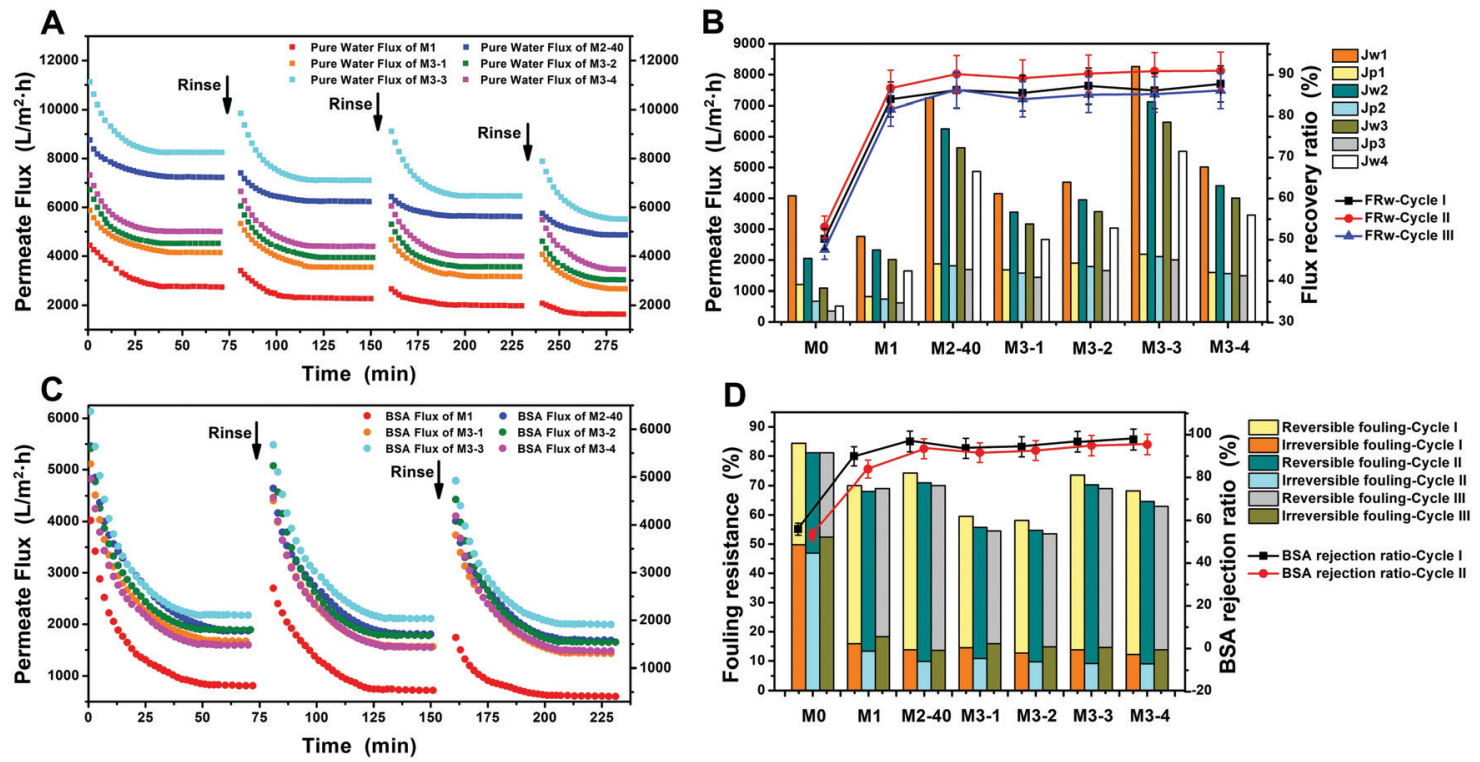

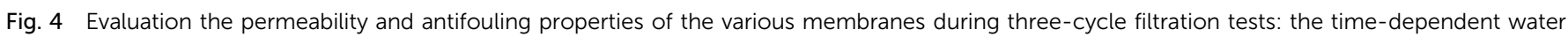

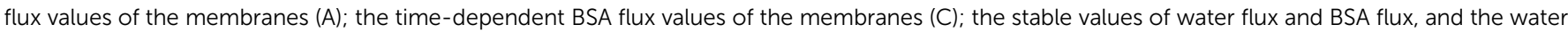

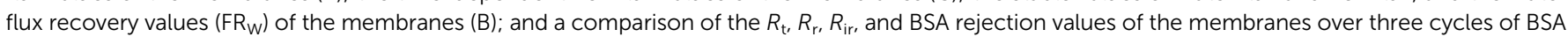
solution filtration measurements (D). 
strategy includes both an anti-adhesion approach and an antibacterial approach. The anti-adhesion approach aims to inhibit the initial adhesion of foulants on membranes, while the antibacterial approach focuses on the inactivation of organisms via interfering with bacterial growth through the introduction of antibacterial materials. To overcome the inherent drawbacks of single active or passive strategies, L-cysteine and AgNPs were both utilized for the modification of membranes to simultaneously acquire active antifouling and passive antibacterial properties. AgNPs are effective at preventing the growth, proliferation, and colonization of microorganisms, and the accompanying membrane biofouling that can occur after long-term operation. Simultaneously, every ion pair made up of positive and negative groups in zwitterionic L-cysteine can bind a vast quantity of water molecules and then form a protective hydration layer on the material surface, and this hydration layer can reduce direct contact between pollutants and the surface dramatically. The

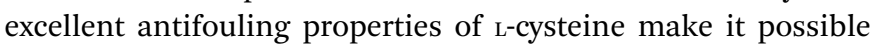
to greatly reduce the initial adhesion of foulants and the deposition of dead bacteria on the membranes. This then contributes to the antifouling activity and triggers a subsequent decrease in bacterial attachment. The greatly enhanced permeability and antifouling properties of the modified membranes, especially M2-40 and M3-3, were demonstrated in these conventional filtration experiments and were further proved via various filtration or adsorption tests. Moreover, the outstanding anti-adhesive and antibacterial properties were also researched in the following bacterial experiments.
Considering the outstanding hydrophilicity of the PVDF/ PDA-AgNPs hybrid membranes and PVDF/PDA-AgNPs-Cys hybrid membranes, self-driven filtration experiments were also carried out to evaluate the permeability of the membranes without external pressure or energy. The experiments were examined over an operation period of $4 \mathrm{~h}$. It was observed that the pristine PVDF membrane begin to permeate water after $1 \mathrm{~h}$ of filtration, but the modified membranes permeated water as soon as filtration began. As shown in Fig. 5A, the pristine PVDF membrane achieved the lowest permeate flux of $8.4 \mathrm{~L} \mathrm{~m}^{-2} \mathrm{~h}^{-1}$, while the values were 40.8, 115.2, 28.8, 48.0, 184.8, and $55.2 \mathrm{~L} \mathrm{~m}^{-2} \mathrm{~h}^{-1}$ for M1, M2-40, and the M3-X series, respectively. It was obvious that the modified membranes all showed superior permeate flux, and the stable value of M3-3 was even more than twenty times that of the pristine PVDF membrane. All these results indicated that the wetting abilities and penetration abilities of the modified membranes were greatly improved, and M2-40 and M3-3 exhibited more outstanding permeability. In our previous work, we proposed that it was the existence of a growing self-driven force after the water flow reached inside the membranes that contributed to the great enhancement of permeability during self-driven filtration experiments.

In view of the excellent permeability and antifouling performances of the functionalized membranes, NOM solution filtration experiments were further conducted to evaluate their practical applications. As shown in Fig. 5B, the stable NOM solution flux ( $J_{\text {NOM }}$ ) values for M0 and the M3-X series were 734.2, 757.3, 798.1, 1537.4, and $937.6 \mathrm{~L} \mathrm{~m}^{-2} \mathrm{~h}^{-1}$, respectively. The NOM flux decreased
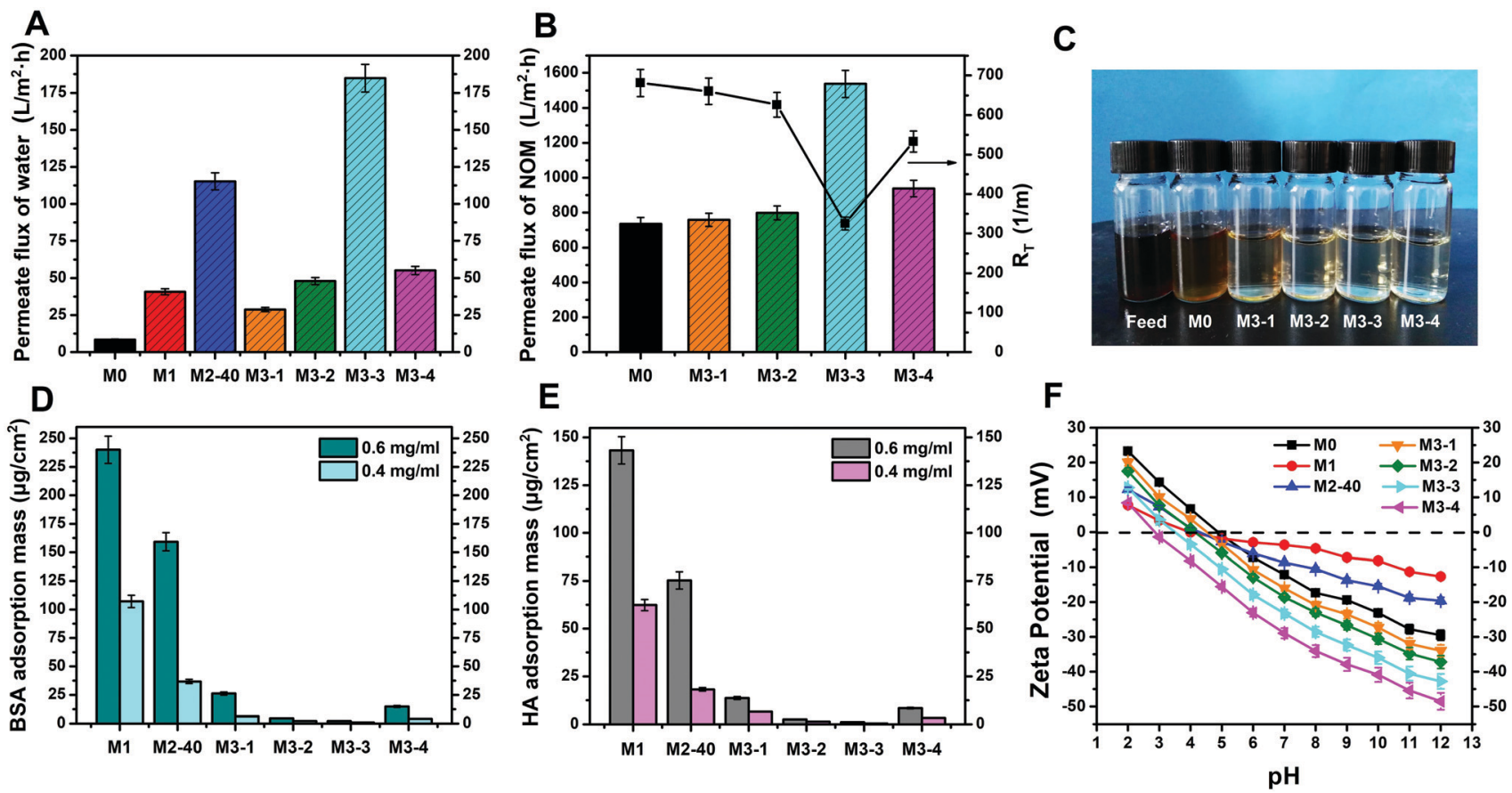

Fig. 5 The stable values of water flux for the membranes during self-driven filtration experiments (A). The stable values of NOM flux for the membranes during NOM solution filtration experiments (B), and a photograph of the feed solution and filtrates taken after filtration tests using the pristine PVDF membrane and PVDF/PDA-AgNPs-Cys hybrid membranes (C). Evaluating the nonspecific protein adsorption properties of various membranes: the static adsorption masses of BSA after exposure to $0.4 \mathrm{~g} \mathrm{~L}^{-1}$ and $0.6 \mathrm{~g} \mathrm{~L}^{-1} \mathrm{BSA}$ solutions for $24 \mathrm{~h}(\mathrm{D})$ and the static adsorption masses of $\mathrm{HA}$ after exposure to $0.4 \mathrm{~g} \mathrm{~L}^{-1}$ and $0.6 \mathrm{~g} \mathrm{~L}^{-1} \mathrm{HA}$ solution for $24 \mathrm{~h}(\mathrm{E})$. The surface zeta potentials of the various membranes at different $\mathrm{pH}$ values (F). 
markedly compared with the water flux and BSA flux in conventional filtration experiments due to the high dynamic viscosity of NOM solutions and the part-blockage of the membrane pores. Besides, the NOM flux values of M3-X, especially M3-3, were all higher than that of M0, suggesting that M3-X showed better antifouling properties toward NOM solutions. The total membrane resistance $\left(R_{\mathrm{T}}\right)$ was also calculated to quantify the antifouling properties of the membranes toward NOM solutions. It was observed that M3-X showed lower $R_{\mathrm{T}}$ values than M0, and M3-3 displayed an $R_{\mathrm{T}}$ value of only $325.2 \mathrm{~m}^{-1}$, further confirming the enhanced antifouling properties of the modified membranes. Fig. 5C shows photographs of the initially prepared NOM solution, and the collected filtrate samples after separation with M0 and M3-X. The color of the initially prepared NOM solution was dark brown, and the color of the collected filtrate after separation with the pristine PVDF membrane faded slightly and changed to brown. However, after separation with the PVDF/PDA-AgNPs-Cys hybrid membranes, the collected filtrate samples changed to being colorless and transparent. These indicated that most of the contaminants were effectively eliminated and the NOM solutions were effectively separated by the modified membranes. As a result, it is believable that the PVDF/PDA-AgNPs-Cys hybrid membranes have greatly promise for use in membrane-based NOM wastewater treatment for practical applications.

Protein static adsorption tests were further researched to assess the nonspecific protein adsorption and protein resistance properties of the membranes. $0.4 \mathrm{~g} \mathrm{~L}^{-1}$ and $0.6 \mathrm{~g} \mathrm{~L}^{-1}$ BSA solution and HA solution were used as test samples. Fig. 5D and $\mathrm{E}$ present the amounts of adsorbed BSA and HA on the membrane surfaces after exposing the test samples for $24 \mathrm{~h}$. Our research displayed that the amounts of adsorbed BSA on the pristine PVDF membrane were $1534.13 \mu \mathrm{g} \mathrm{cm}^{-2}$ and $1821.65 \mu \mathrm{g} \mathrm{cm}{ }^{-2}$, and the adsorbed HA amounts were $870.67 \mu \mathrm{g} \mathrm{cm}^{-2}$ and $986.67 \mu \mathrm{g} \mathrm{cm}^{-2}$, respectively. In the presence of PDA microspheres and AgNPs, the degree of nonspecific protein adsorption decreased significantly. In particular, for PVDF/PDA-AgNPs-Cys hybrid membranes, the adsorption values for BSA and HA both reduced dramatically, and hardly any adsorbed BSA or HA was observed on the M3-2 and M3-3 surfaces. The adsorption values of M3-3 were 99\% lower than those of the pristine PVDF membrane. All these results indicated that the modified membranes could effectively inhibit the adsorption of BSA and HA pollutants, especially M3-X, with excellent active-passive integrated antifouling and antibacterial properties.

Considering the fact that surface charge characteristics are a crucial factor determining the antifouling performance of membranes, zeta potential measurements were also carried out in the $\mathrm{pH}$ range from 2 to 12 . As shown in Fig. 5F, all the membranes exhibited positive charge characteristics at relatively acidic $\mathrm{pH}$ values and this then altered to negative charge characteristics upon increasing the $\mathrm{pH}$. This was ascribed to the preferential adsorption of cations $\left(\mathrm{K}^{+}, \mathrm{Na}^{+}\right.$, or $\left.\mathrm{H}^{+}\right)$at acidic $\mathrm{pH}$ or the adsorption of anions $\left(\mathrm{Cl}^{-}\right.$or $\left.\mathrm{OH}^{-}\right)$at alkaline $\mathrm{pH}$ on the membrane surfaces. The surface zeta potential of M0 was positive at $\mathrm{pH}$ values from 2 to 5 and was negative as the $\mathrm{pH}$ changed from 5 to 12 . M1 and M2-40 showed positive charge characteristics in a
$\mathrm{pH}$ range from 2 to 4.3 and were negative in the $\mathrm{pH}$ range of 4.3 to 12. Moreover, the absolute positive/negative zeta potential values for M1 and M2-40 were significantly lower than that of the pristine PVDF membrane, which could be attributed to the retardation of the adsorption rates of anions or cations on the membrane surface due to the hydrophilic PDA and AgNPs. For the PVDF/ PDA-AgNPs-Cys hybrid membranes, the isoelectric points further decreased, and the absolute values of the negative zeta potentials were all higher than those of the pristine PVDF membrane due to the amphoteric nature of L-cysteine. This was favorable for the great enhancement of the antifouling properties, especially for M3-3 and M3-4.

\subsection{Antibacterial properties of the hybrid membranes}

AgNPs were reported to be a nonspecific antibacterial agent and they have exhibited excellent antibacterial properties in many areas. In this work, both $E$. coli and $S$. aureus were used in diffusion inhibition zone (DIZ) tests to evaluate the antibacterial properties of the membranes. As shown in Fig. 6, it could be observed that the two kinds of bacteria grew well around M0 and M1, while clear inhibition zones occurred at the outer edges of the M2-X and M3-X series. This indicated that M0 and M1 did not show any antibacterial properties, while M2-X and M3-X showed great antibacterial properties. This could be attributed to the effective antibacterial properties of AgNPs. Generally speaking, AgNPs are believed to act as an antibacterial agent either upon contact with the bacteria or via releasing ions, which can attack bacteria via disturbing their function and preventing their growth. As a result, apparent inhibition zones were formed around all the silver-modified membranes. Otherwise, the antibacterial properties of the membranes could be qualitatively evaluated via measuring the diameters of the inhibition zones, and the data are shown in Table 2. It was observed that M2-40 exhibited the best antibacterial properties among the M2-X series due to it having the most AgNPs immobilized on the PVDF/PDA-AgNPs hybrid membrane. In addition, the PVDF/PDA-AgNPs-Cys hybrid membranes also showed good inhibition zones, indicating that covalently attached L-cysteine had only a small adverse impact on the antibacterial properties of the membranes.

The colony forming unit (CFU) counting method was also employed to assess the antibacterial properties of the membranes against $E$. coli and $S$. aureus. As shown in Fig. 7, hundreds of CFUs were observed on LB agar plates with the bacterial suspension solutions obtained from M0 and M1, while only a small number of CFUs was observed for M2-X and M3-X, and no CFUs were seen for M2-40, M3-1, and M3-2. The amounts of attached live $E$. coli and $S$. aureus on the agar plates were normalized on the basis of M0, and the data are exhibited in Fig. S4 (ESI $\dagger$ ). It was confirmed that the amounts of live E. coli and S. aureus decreased by $3.98 \%$ and $32.14 \%$ in the case of M1, indicating that the PDA microspheres had very little bacterial killing activity against $E$. coli and a certain level of antibacterial properties against $S$. aureus. M2-X and M3-X almost all displayed reductions in the amounts of live $E$. coli and $S$. aureus of over $90 \%$, and the bacterial viabilities relating to M2-40, M3-1, and M3-2 were even reduced to $0.00 \%$. All these results showed that AgNPs imparted the PVDF/PDA-AgNPs hybrid 

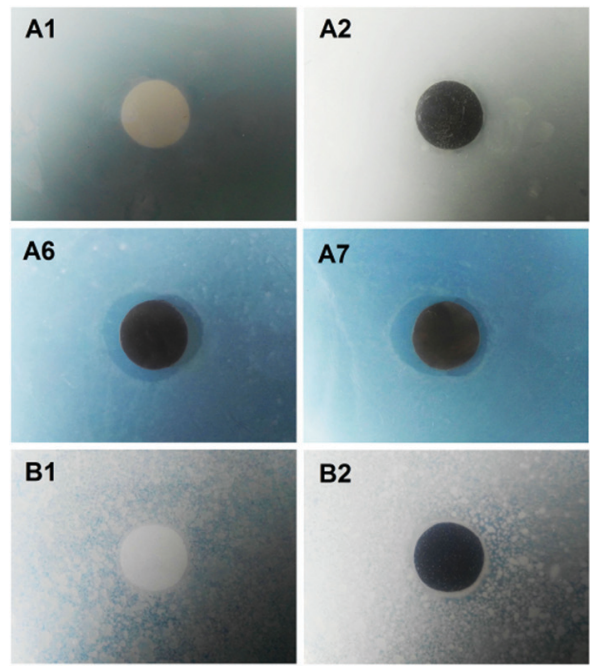

B2

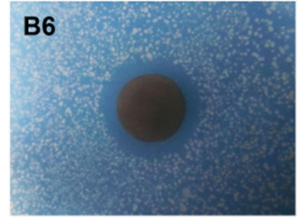

B7

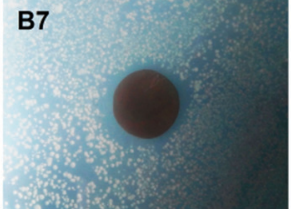

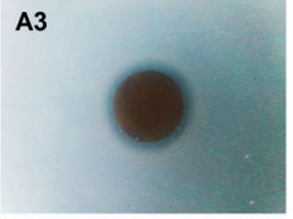

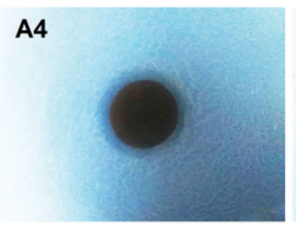

A9
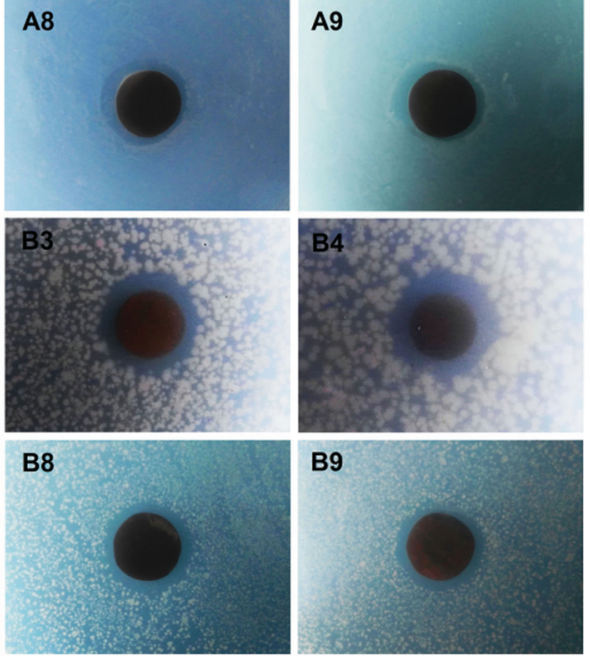

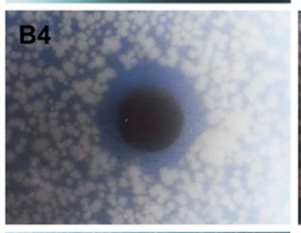

B9

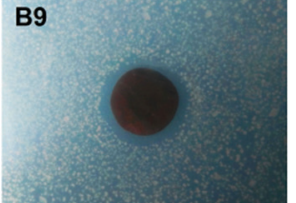

A5
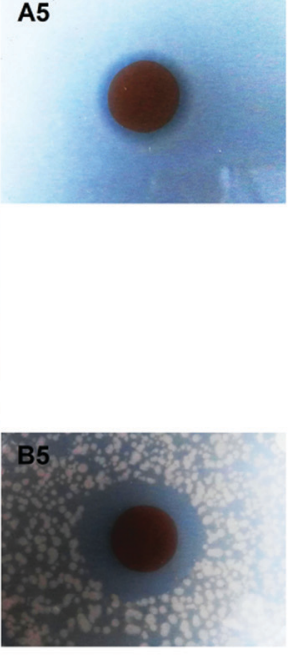

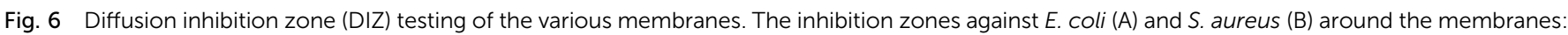

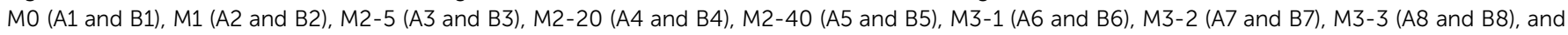
M3-4 (A9 and B9).

Table 2 The measured diameters of the inhibition zones from DIZ tests

\begin{tabular}{lllll}
\hline & \multicolumn{5}{c}{ The diameter of the inhibition zone (mm) } \\
\cline { 2 - 5 } Membrane & E. coli & S. aureus & $\begin{array}{l}\text { E. coli (after Ag } \\
\text { leaching tests) }\end{array}$ & $\begin{array}{l}\text { S. aureus (after } \\
\text { Ag leaching tests) }\end{array}$ \\
\hline M0 & 13.0 & 13.0 & - & - \\
M1 & 13.0 & 13.0 & - & - \\
M2-5 & 16.2 & 19.3 & 15.9 & 19.1 \\
M2-20 & 16.5 & 22.4 & 16.3 & 22.0 \\
M2-40 & 17.0 & 24.8 & 16.7 & 24.5 \\
M3-1 & 19.2 & 20.0 & - & - \\
M3-2 & 18.5 & 17.6 & - & - \\
M3-3 & 17.2 & 17.3 & - & - \\
M3-4 & 17.0 & 17.1 & - & -
\end{tabular}

membranes and PVDF/PDA-AgNPs-Cys hybrid membranes with outstanding antibacterial properties. It is also further demonstrated that the in situ formation of AgNPs on the membranes through the reduction of $\mathrm{Ag}^{+}$by PDA microspheres was proven to be an efficient method for generating excellent antibacterial properties in the field of membranes.

As is well known, bacteria are another kind of contaminant that can adhere onto membranes, thus resulting in severe biological contamination. In this work, we chose $E$. coli as a typical type of bacteria to evaluate the anti-adhesive properties of the membranes. Fig. 8 shows that there was a large number of bacteria attached to the pristine PVDF membrane after $24 \mathrm{~h}$ of incubation. The number of bacteria on the M1 surface displayed a slight decrease, indicating that the PDA microspheres had no effect or led to a modest decrease in bacterial attachment. In contrast, the adhesion of bacteria was significantly suppressed on M2-20 and M2-40, and the amounts of bacterial adhesion were only $15 \%$ to $20 \%$ that of M0. It was the deposition of AgNPs that improved the anti-adhesive properties of the membranes. For the PVDF/PDA-AgNPs-Cys hybrid membranes, smaller amounts of bacteria could be observed on the surfaces, indicating that adhesion was inhibited almost completely. This confirmed that L-cysteine played a critical role in the enhancement of the anti-adhesive properties of the membranes due to the excellent antifouling performance. All these results demonstrated that the anti-adhesive properties of the PVDF/PDA-AgNPs hybrid membranes and PVDF/PDA-AgNPs-Cys hybrid membranes were considerably enhanced, and they maintained durable resistance. We believe it was a combined effect of AgNPs and L-cysteine that imparted the membranes with outstanding antiadhesive properties.

\subsection{Stability of the immobilized AgNPs on the membranes}

To test the stability and release behavior of the immobilized AgNPs on the membranes, Ag leaching tests were performed over one month, simulating sink conditions. Fig. 9C and D show the release rates of silver in the first 7 days and the accumulated silver amounts over 30 days. The initial release rates were approximately $0.1434,0.1854$, and $0.2367 \mu \mathrm{g} \mathrm{cm} \mathrm{cday}^{-1}$ for M2-5, M2-20, and M2-40, respectively. On the 7th day, the values were separately reduced to $0.0415,0.0615$, and $0.0789 \mu \mathrm{g} \mathrm{cm}^{-2}$ day $^{-1}$. The corresponding accumulation data shown in Fig. 9D exhibit that the amounts of accumulated silver increased linearly in the first 13-18 days, and then reached relatively stable values. This revealed that there was continuous silver release into the solution in the first 13-18 days, after 

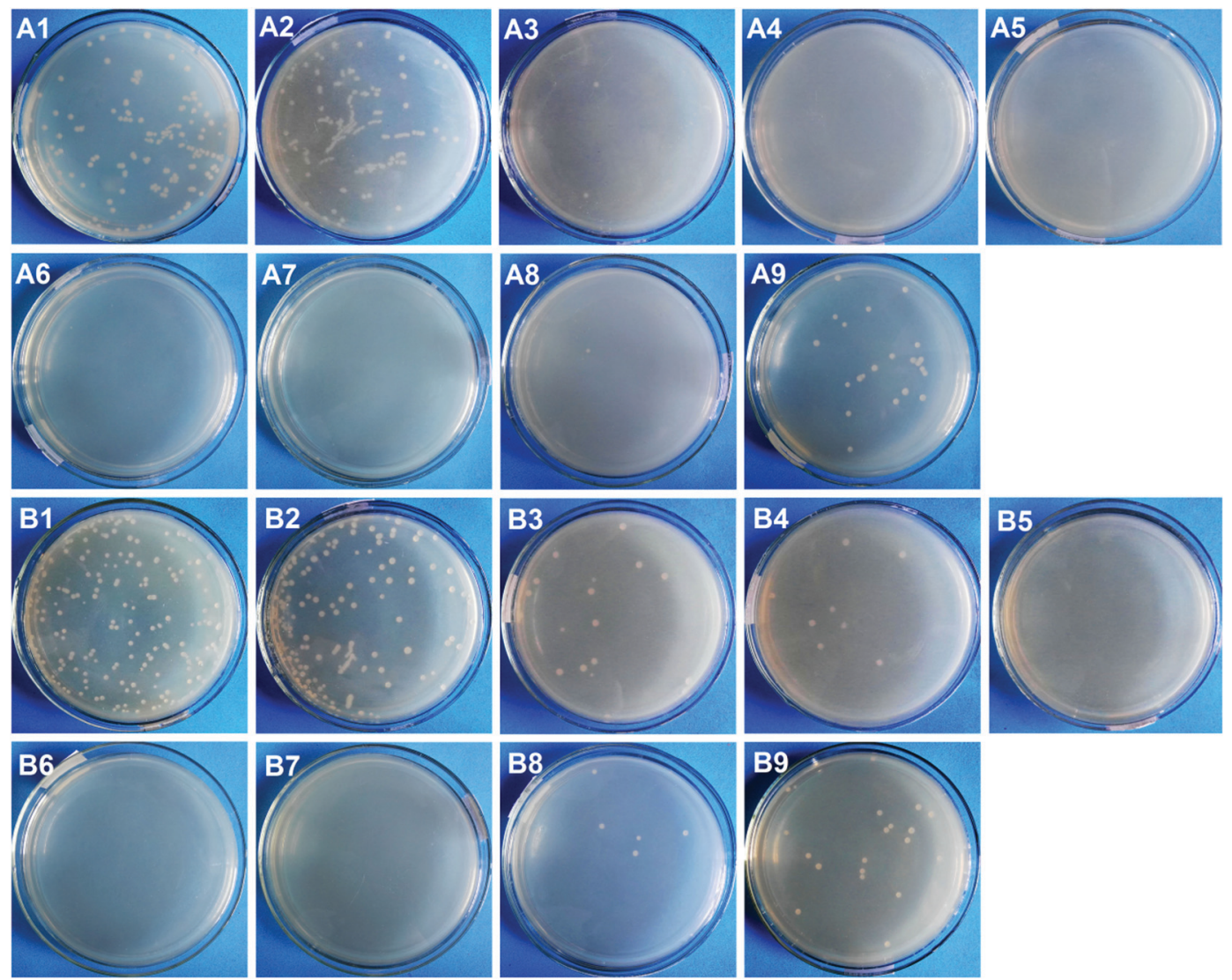

Fig. 7 The colony forming unit (CFU) inhibition testing of the various membranes. E. coli colonies (A) and S. aureus colonies (B) on LB agar plates: MO (A1 and B1), M1 (A2 and B2), M2-5 (A3 and B3), M2-20 (A4 and B4), M2-40 (A5 and B5), M3-1 (A6 and B6), M3-2 (A7 and B7), M3-3 (A8 and B8), and M3-4 (A9 and B9).
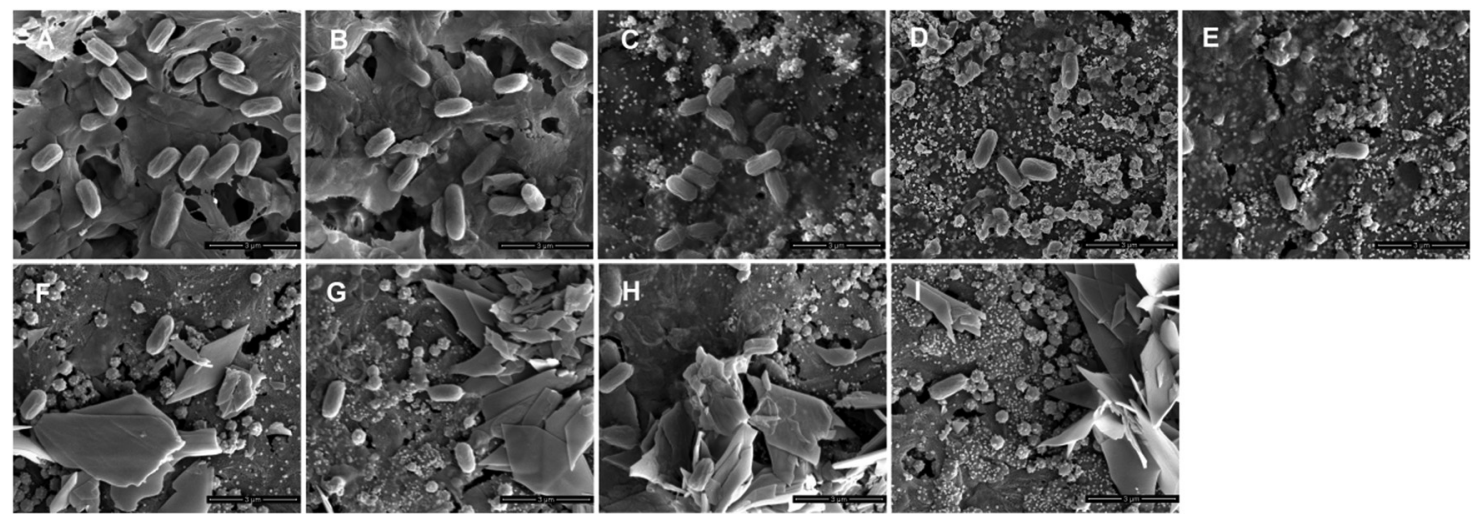

Fig. 8 The adhesion of $E$. coli on various membranes. SEM images of the different membranes: MO (A), M1 (B), M2-5 (C), M2-20 (D), M2-40 (E), M3-1 (F), M3-2 (G), M3-3 (H), and M3-4 (I).

which little additional silver release was observed. The total released amounts of silver into water over 30 days were 0.7232 , 1.0521, and $1.3612 \mu \mathrm{g} \mathrm{cm}^{-2}$ for M2-5, M2-20, and M2-40, respectively. The corresponding solution concentrations were calculated to be $19.20,27.93$, and $36.14 \mu \mathrm{g} \mathrm{L} \mathrm{L}^{-1}$, which are significantly lower than the maximum contaminant limit of silver in drinking water (i.e., $100 \mu \mathrm{g} \mathrm{L}^{-1}$ ) set by U.S. Environmental Protection Agency regulations ${ }^{48}$ and World Health Organization guidelines. ${ }^{49}$ Therefore, there would likely be no risk to health if the membranes were applied to water filtration. The low released amounts of silver indicated that AgNPs were immobilized firmly on the membranes, which can be mostly attributed to the guiding reduction effect from the PDA microspheres on $\mathrm{Ag}^{+}$. The reason was that not only can the $\mathrm{O}$ sites and $\mathrm{N}$ sites of PDA serve as anchors for guiding the immobilization of AgNPs on PDA microspheres, but also the phenolic hydroxyl groups distributed 


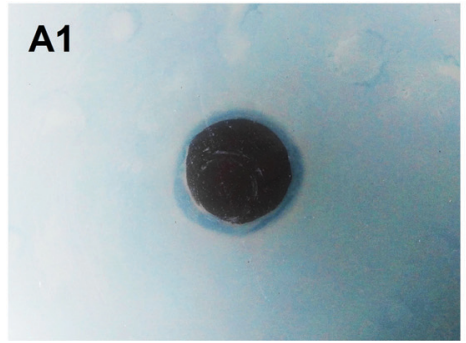

A2
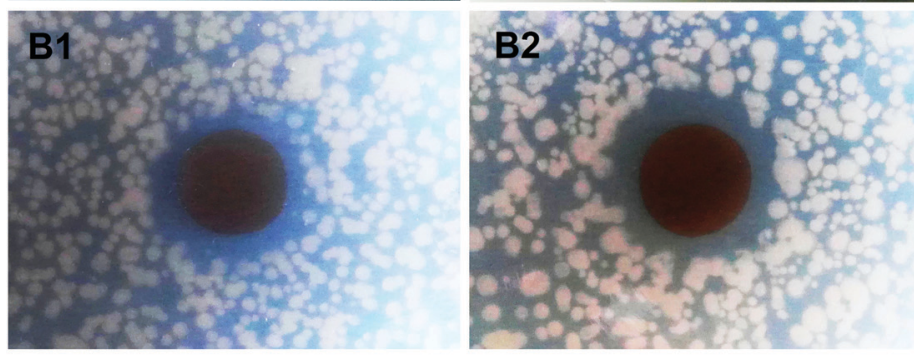

A3

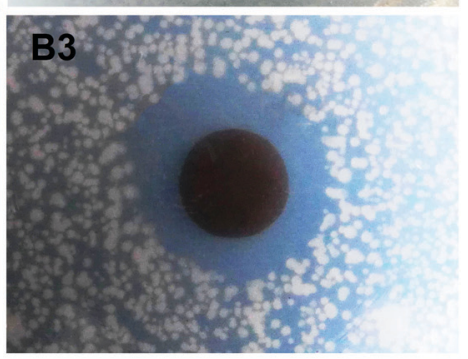

C

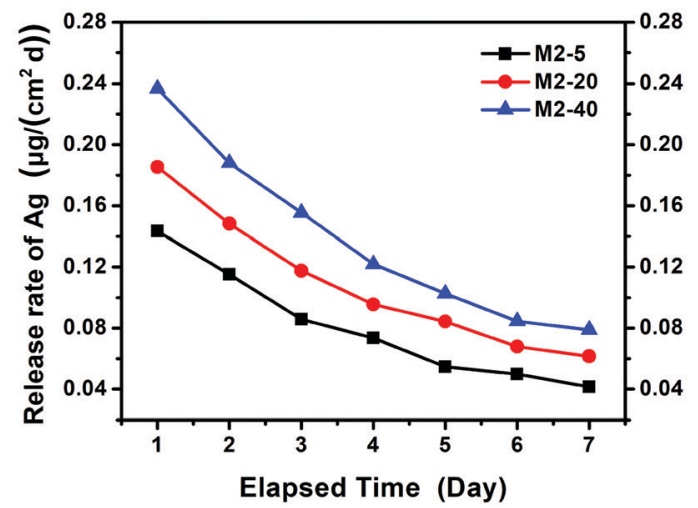

D

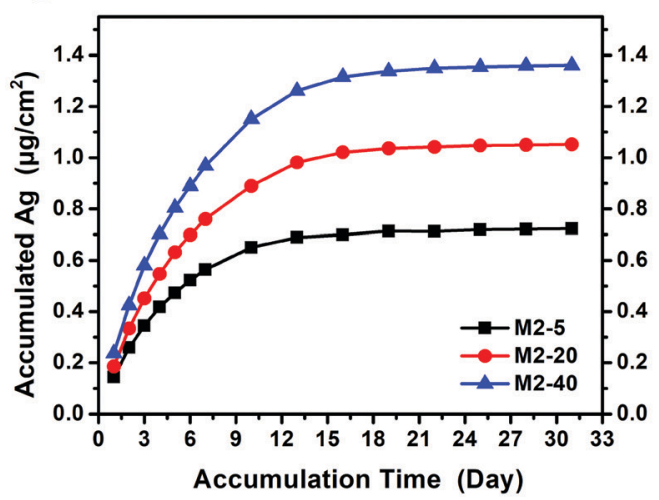

Fig. 9 The diffusion inhibition zone (DIZ) testing of the PVDF/PDA-AgNPs hybrid membranes after Ag leaching tests. The inhibition zones of E. coli (A) and S. aureus (B) around the membranes: M2-5 (A1 and B1), M2-20 (A2 and B2), and M2-40 (A3 and B3). The stabilities of AgNPs on the PVDF/PDAAgNPs hybrid membranes: the release rates of silver in the first 7 days (C), and the accumulated silver amounts over 30 days (D).

in all directions on the PDA microspheres are more conducive to immobilizing AgNPs than the phenolic hydroxyl groups distributed in certain directions from the thin adhering PDA flat layer on the membrane. The good stability of the immobilized AgNPs on the membranes make it possible for the modified membranes to operate in aqueous environments over long timescales and they also have great potential for showing long-lasting antibacterial activities.

Additionally, DIZ tests were also carried out to evaluate the antibacterial properties of the PVDF/PDA-AgNPs hybrid membranes after Ag leaching tests. As shown in Fig. 9A and B, apparent inhibition zones were observed toward both $E$. coli and $S$. aureus, and the diameters of the inhibition zones were comparable to those of freshly prepared PVDF/PDA-AgNPs hybrid membranes without $\mathrm{Ag}$ leaching tests, as shown in Table 2. This indicated that the dissolution and loss of AgNPs occurred at low levels, and the immobilized AgNPs on the membranes could impart the membranes with long-lasting antibacterial activities. This result corresponded well with the above-mentioned stability and release behavior of the immobilized AgNPs on the membranes.

\section{Conclusions}

A novel and versatile active-passive integrated strategy to integrate both antifouling and antibacterial properties into PVDF membranes has been developed through utilizing the versatile features of PDA microspheres, AgNPs, and L-cysteine. The effects of modification on the morphologies, hydrophilicity, permeability, antifouling properties, antibacterial properties, and long-term stability of the various prepared hybrid membranes were investigated in detail. Several findings could be concluded, as follows:

(1) The PDA microspheres on the modified membranes served as primers, and we proposed that a "PDA guiding effect" exists during the modification process. Specifically speaking, it was a guiding reduction effect from PDA microspheres towards $\mathrm{Ag}^{+}$and a guiding generation effect towards $\mathrm{L}$-cysteine that worked separately in favor of the generation of AgNPs and cysteine flowers in the substrate interior, therefore endowing the membranes with excellent properties.

(2) The active-passive integrated strategy presented in this research simultaneously includes both an anti-adhesion approach for reducing the initial adsorption of organisms and an anti-bacterial 
approach for suppressing the activities of attached organisms on the membranes. Conventional filtration experiments clearly demonstrated that the zwitterionic characteristics of L-cysteine made it possible to greatly reduce the initial adhesion of foulants and the deposition of dead bacteria on the membranes. Various bacteria-based experiments proved that AgNPs were effective at preventing the growth, proliferation, and colonization of microorganisms, as well as accompanying membrane biofouling after long-term operation.

(3) NOM solution filtration experiments and protein adsorption tests were also carried out to evaluate the practical applications of the modified membranes. These results showed that most of the NOM and protein contaminants were effectively removed, and the contaminant solutions were effectively filtered by the modified membranes. As a result, it is believed that the PVDF/PDA-AgNPsCys hybrid membranes have great promise for use in membranebased NOM wastewater treatment for practical applications.

\section{Conflicts of interest}

There are no conflicts to declare.

\section{Acknowledgements}

Financial support from the Natural Science Foundation of Fujian Province (No. 83019022) and the National Natural Science Foundation of China (No. 51303028) is acknowledged. Test fund support by Test Center of Fuzhou University (2020T021).

\section{References}

1 C. Castel and E. Favre, J. Membr. Sci., 2018, 548, 345-357.

2 W. J. Lau and A. P. Ríos, Chem. Eng. Technol., 2018, 41, 210. 3 J. M. Dickhout, J. Moreno, P. M. Biesheuvel, L. Boels, R. G. H. Lammertink and W. M. de Vos, J. Colloid Interface Sci., 2017, 487, 523-534.

4 J. Kujawa, S. Cerneaux, S. Koter and W. Kujawski, ACS Appl. Mater. Interfaces, 2014, 6, 14223-14230.

5 S. Qavi, A. P. Lindsay, M. A. Firestone and R. Foudazi, J. Membr. Sci., 2019, 580, 125-133.

6 S. Rajesh, S. Schneiderman, C. Crandall, H. Fong and T. J. Menkhaus, ACS Appl. Mater. Interfaces, 2017, 9, 41055-41065.

7 S. N. Rashkeev and B. Shomar, Environ. Res., 2020, 190, 110000.

8 A. Bogler, S. H. Lin and E. B. Zeev, J. Membr. Sci., 2017, 542, 378-398.

9 A. F. Faria, C. H. Liu, M. Xie, F. Perreault, L. D. Nghiem, J. Ma and M. Elimelech, J. Membr. Sci., 2017, 525, 46-156.

10 S. T. Weinman, M. Bass, S. Pandit, M. Herzberg, V. Freger and S. M. Husson, J. Membr. Sci., 2018, 548, 490-501.

11 M. M. Zhu, Y. Fang, Y. C. Chen, Y. Q. Lei, L. F. Fang, B. K. Zhu and H. Matsuyama, J. Colloid Interface Sci., 2021, 584, 225-235.

12 H. Lee, S. M. Dellatore, W. M. Miller and P. B. Messersmith, Science, 2007, 318, 426-430.
13 J. H. Waite and X. X. Qin, Biochemistry, 2001, 40, 2887-2893.

14 D. R. Dreyer, D. J. Miller, B. D. Freeman, D. R. Paul and C. W. Bielawski, Chem. Sci., 2013, 4, 3796-3802.

15 D. H. Liang, Z. Lu, H. Yang, J. T. Gao and R. Chen, ACS Appl. Mater. Interfaces, 2016, 8, 3958-3968.

16 M. S. Khan, H. Ameer, A. Ali, Y. Y. Li, L. Yang, X. Ren and Q. Wei, Biosens. Bioelectron., 2020, 162, 112235.

17 M. S. Haider, G. N. Shao, S. M. Imran, S. S. Park, N. Abbas, M. S. Tahir, M. Hussain, W. Bae and H. T. Kim, Mater. Sci. Eng., C, 2016, 62, 732-745.

18 T. A. Makhetha and R. M. Moutloali, J. Membr. Sci., 2021, 618, 118733.

19 C. X. Dong, Z. Wang, J. H. Wu, Y. Wang, J. X. Wang and S. C. Wang, Desalination, 2017, 401, 32-41.

20 P. Biswas and R. Bandyopadhyaya, J. Colloid Interface Sci., 2017, 491, 13-26.

21 A. Behboudi, Y. Jafarzadeh and R. Yegani, J. Environ. Chem. Eng., 2018, 6, 1764-1773.

22 Z. H. Xu, S. J. Ye, G. L. Zhang, W. B. Li, C. J. Gao, C. Shen and Q. Meng, J. Membr. Sci., 2016, 509, 83-93.

23 M. R. He, K. Gao, L. J. Zhou, Z. W. Jiao, M. Y. Wu, J. L. Cao, X. D. You, Z. Y. Cai, Y. L. Su and Z. Y. Jiang, Acta Biomater., 2016, 40, 142-152.

24 N. C. H. Vy, C. D. Liyanage, R. M. L. Williams, J. M. Fang, P. M. Kerns, H. C. Schniepp and D. H. Adamson, Macromolecules, 2020, 53, 10278-10288.

25 H. J. Yue, H. J. Sun, T. J. Peng, B. Liu and Y. Y. Xie, J. Mol. Struct., 2018, 1163, 449-454.

26 Y. F. Wang, Y. Xiao, G. R. Gao, J. Chen, R. X. Hou, Q. Wang, L. Liu and J. Fu, J. Mater. Chem. B, 2017, 5, 511-516.

27 H. J. Zhang, G. C. Li, L. Deng, H. X. Zeng and Z. Shi, J. Colloid Interface Sci., 2019, 543, 183-191.

28 B. N. Ganguly, B. Maity, T. K. Maity, J. Manna, M. Roy, M. Mukherjee, S. Debnath, P. Saha, N. Shilpa and R. K. Rana, Langmuir, 2018, 34, 1447-1456.

29 S. Azari and L. Zou, Desalination, 2013, 324, 79-86.

30 Y. H. Liao, R. Yuan, Y. Q. Chai, Y. Zhuo and X. Yang, Anal. Biochem., 2010, 402, 47-53.

31 J. H. Li, X. X. Ni, D. B. Zhang, H. Zheng, J. B. Wang and Q. Q. Zhang, Appl. Surf. Sci., 2018, 444, 672-690.

32 J. H. Jiang, P. B. Zhang, L. P. Zhu, B. K. Zhu and Y. Y. Xu, J. Mater. Chem. B, 2015, 3, 7698-7706.

33 A. Qin, X. Li, X. Z. Zhao, D. P. Liu and C. J. He, ACS Appl. Mater. Interfaces, 2015, 7, 8427-8436.

34 J. H. Jiang, L. P. Zhu, H. T. Zhang, B. K. Zhu and Y. Y. Xu, J. Membr. Sci., 2014, 457, 73-81.

35 X. Z. Zhao, H. X. Xuan, Y. L. Chen and C. J. He, J. Membr. Sci., 2015, 494, 48-56.

36 B. Wu, F. Hochstrasser, E. Akhondi, N. Ambauen, L. Tschirren, M. Burkhardt, A. G. Fane and W. Pronk, Water Res., 2016, 93, 133-140.

37 J. Q. Wang, H. Guo, Z. Yang, Y. Mei and C. Y. Tang, J. Membr. Sci., 2017, 525, 298-303.

38 R. Shevate, M. Kumar, M. Karunakaran, M. N. Hedhili and K. V. Peinemann, J. Membr. Sci., 2017, 529, 185-194. 
39 M. Schulz, A. Soltani, X. Zheng and M. Ernst, J. Membr. Sci., 2016, 507, 154-164.

40 X. Z. Zhao, Y. L. Chen, H. X. Xuan and C. J. He, New J. Chem., 2016, 40, 441-446.

41 M. Khajouei, M. Jahanshahi and M. Peyravi, J. Taiwan Inst. Chem. Eng., 2018, 85, 237-247.

42 Y. Orooji, M. Faghih, A. Razmjou, J. W. Hou, P. Moazzam, N. Emami, M. Aghababaie, F. Nourisfa, V. Chen and W. Q. Jin, Carbon, 2017, 111, 689-704.

43 Z. Yang, D. Saeki and H. Matsuyama, J. Membr. Sci., 2018, 550, 332-339.
44 Z. Y. Liu and Y. X. Hu, ACS Appl. Mater. Interfaces, 2016, 8, 21666-21673.

45 V. Ball, I. Nguyen, M. Haupt, C. Oehr, C. Arnoult, V. Toniazzo and D. Ruch, J. Colloid Interface Sci., 2011, 364, 359-365.

46 L. Bekale, S. Barazzouk and S. Hotchandani, Part. Part. Syst. Charact., 2014, 31, 843-850.

47 P. F. Li, X. M. Cai, D. Wang, S. C. Chen, J. Yuan, L. Li and J. Shen, Colloids Surf., B, 2013, 110, 327-332.

48 H. T. Ratte, Environ. Toxicol. Chem., 1999, 18, 89-108.

49 M. Y. Zhang, R. W. Field and K. S. Zhang, J. Membr. Sci., 2014, 471, 274-284. 